ANALYSIS \& PDE Volume $5 \quad$ No. $5 \quad 2012$ (1)

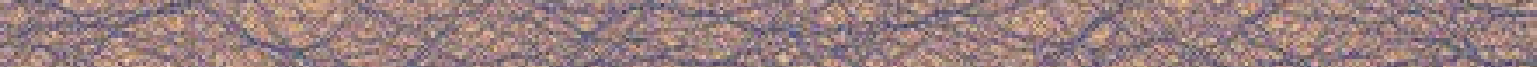
2. SUNHI CHOI AND INWON KIM 3 ;N THE TWO-PHASE STEFAN PROBLEM: REGULARIZATION NEAR LIPSCHITZ INITIAL DATA BY PHASE DYNAMICS 


\title{
THE TWO-PHASE STEFAN PROBLEM: REGULARIZATION NEAR LIPSCHITZ INITIAL DATA BY PHASE DYNAMICS
}

\author{
SunHI CHOI AND INWON KIM
}

In this paper we investigate the regularizing behavior of two-phase Stefan problem near initial Lipschitz data. A description of the regularizing phenomena is given in terms of the corresponding space-time scale.

\section{Introduction}

Consider $u_{0}(x): B_{R}(0) \rightarrow \mathbb{R}$ with $R \gg 1$ and $u_{0} \geq-1,\left|\left\{u_{0}=0\right\}\right|=0$ and $u_{0}(x)=-1$ on $\partial B_{R}(0)$ (see Figure 1). The two-phase Stefan problem can be formally written as

$$
\begin{cases}u_{t}-\Delta u=0 & \text { in }\{u>0\} \cup\{u<0\}, \\ u_{t} /\left|D u^{+}\right|=\left|D u^{+}\right|-\left|D u^{-}\right| & \text {on } \partial\{u>0\}, \\ u(\cdot, 0)=u_{0}, & \text { on } \partial B_{R}(0) .\end{cases}
$$

Here $D u$ denotes the spatial derivative of $u$, and $u^{+}$and $u^{-}$respectively denote the positive and negative parts of $u$, i.e,

$$
u^{+}:=\max (u, 0) \quad \text { and } \quad u^{-}:=-\min (u, 0)
$$

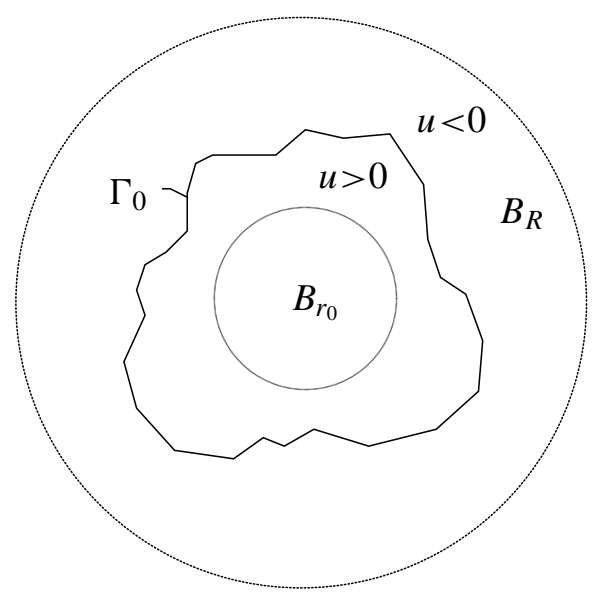

Figure 1. Initial setting of the problem.

I. Kim is partially supported by NSF grant number 0970072 .

MSC2010: 35K55, 35R35, 76D27.

Keywords: free boundary problem, Stefan problem, phase transition, regularity near initial data, viscosity solutions. 
The classical Stefan problem describes the phase transition between solid/liquid or liquid/liquid interface (see [Meirmanov 1992; Oleinik et al. 1993]). In our setting, we consider a bounded domain $\Omega_{0} \subset B_{R}(0)$ and initial data $u_{0}(x)$ such that

$$
\left\{u_{0}>0\right\}=\Omega_{0} \quad \text { and } \quad\left\{u_{0}<0\right\}=B_{R}(0)-\bar{\Omega}_{0} .
$$

To avoid complications at infinity, we consider the problem in the domain $Q=B_{R}(0) \times[0, \infty)$. For simplicity we have set $u=-1$ on $\partial B_{R}(0)$; our analysis presented in this paper applies to (ST2) with the generalized Dirichlet condition

$$
u=f(x, t)<0 \quad \text { on } \partial B_{R}(0),
$$

where $f(x, t)$ is smooth.

Since our initial data will be only locally Hölder continuous, we employ the notion of viscosity solutions to discuss the evolution of the problem. Viscosity solutions for (ST2) were originally introduced by Athanasopoulos et al. [1996] (see also [Caffarelli and Salsa 2005]). As for existence and uniqueness of viscosity solutions for (ST2), we refer to [Kim and Požár 2011].

Note that the second condition of (ST2) states that the normal velocity $V_{x, t}$ at each free boundary point $(x, t) \in \partial\{u>0\}$ is given by

$$
V_{x, t}=\left(\left|D u^{+}\right|-\left|D u^{-}\right|\right)(x, t)=\left(D u^{+}(x, t)-D u^{-}(x, t)\right) \cdot v_{x, t},
$$

where $v_{x, t}$ denotes the spatial unit normal vector of $\partial\{u>0\}$ at $(x, t)$, pointing inward with respect to the positive phase $\{u>0\}$.

In this paper we investigate the regularizing behavior of the free boundary $\partial\{u>0\}$. Our main result states that when $\Gamma_{0}:=\partial\left\{u_{0}>0\right\}$ is locally a Lipschitz graph with small Lipschitz constant, then the free boundary immediately regularizes and becomes smooth after $t=0$. Moreover we provide a natural space-time scale for such regularization. More precisely, for $x_{0} \in \Gamma_{0}$, we show that the free boundary regularizes in $B_{d}\left(x_{0}\right)$ by the time $t\left(x_{0}, d\right)$ given in (1-3) (see Theorem 1.1, and also the heuristic discussion below (1-3)). Corresponding results have been obtained in recent studies on the one-phase free boundary problems [Choi et al. 2007; 2009; Choi and Kim 2010], but the presence of two phases poses new challenges in the analysis. For example there is no generic class of global solutions other than radial solutions where topological changes are ruled out. In the one-phase setting we relied on the fact that solutions with star-shaped initial data stay star-shaped over time: this is no longer true in the two-phase setting (see Remark 3.2). More importantly, the interface motion is no longer monotone and competition between positive and negative fluxes across the free boundary necessitates additional localization procedure (see the remarks below Theorem 1.1).

The celebrated results of [Athanasopoulos et al. 1996; 1998] state that if the solution of (ST2) stays close to a Lipschitz profile in the unit space-time neighborhood $B_{1}(0) \times[0,1]$, then the solution is indeed smooth in half of the neighborhood, that is, in $B_{1 / 2}(0) \times[1 / 2,1]$. The main step in our analysis is to prove that the free boundary $\partial\{u>0\}$ stays close to a locally Lipschitz profile in any given scale. Proving this step corresponds to the derivation of several Harnack-type inequalities for our problem, which are of independent interest. 
Before discussing our result in detail, let us introduce precise conditions on the initial data.

(I-a) $\Omega_{0}$ and $u_{0}$ are star-shaped with respect to a ball $B_{r_{0}}(0) \subset \Omega_{0}$.

Observe that then the Lipschitz constant $L$ of $\partial \Omega_{0}$ is determined by $r_{0}$ and $d_{0}$, where

$$
d_{0}:=\sup \left\{\operatorname{dist}\left(x, B_{r_{0}}(0)\right): x \in \partial \Omega_{0}\right\} .
$$

In other words, there exist $h=h\left(r_{0}\right)$ and $L=L\left(r_{0}, d_{0}\right)$ such that for any $x_{0} \in \partial \Omega_{0}$, after rotation of coordinates, one has the representation

$$
B_{h}\left(x_{0}\right) \cap \Omega_{0}=\left\{\left(x^{\prime}, x_{n}\right): x^{\prime} \in \mathbb{R}^{n-1}, x_{n} \leq f(x)\right\},
$$

where $f$ is a Lipschitz function with $\operatorname{Lip} f \leq L$. For simplicity, we set $h=1$.

For a locally Lipschitz domain such as $\Omega_{0}$, there exist growth rates $0<\beta<1<\alpha$ such that the following holds: Let $H$ be a positive harmonic function in $\Omega_{0} \cap B_{2}(x), x \in \partial \Omega_{0}$, with Dirichlet condition on $\partial \Omega_{0} \cap B_{2}(x)$, and with value 1 at $x-e_{n}$. (Here $e_{n}$ is the direction of the axis for the Lipschitz graph near $x$.) Then for $x-s e_{n} \in \Omega_{0} \cap B_{1}(x)$,

$$
s^{\alpha} \leq H\left(x-s e_{n}\right) \leq s^{\beta} .
$$

Below we list conditions on the range of the Lipschitz constant $L$ of the initial positive phase $\Omega_{0}$.

(I-b) $L<L_{n}$ for a sufficiently small dimensional constant $L_{n}$ so that

$$
5 / 6 \leq \beta<\alpha \leq 7 / 6 .
$$

The remaining conditions are on the regularity of $u_{0}$.

(I-c) $-N_{0} \leq \Delta u_{0} \leq N_{0}$ in $\Omega_{0} \cup\left(B_{R}(0)-\bar{\Omega}_{0}\right)$, where $N_{0}$ is some constant.

(I-d) For $x \in \partial \Omega_{0}$, we may let $e_{n}=x /|x|$ after a rotation. Then for small $s>0$ (for $0<s<1 / 10$ ),

$$
\left|D u_{0}\left(x \pm s e_{n}\right)\right| \geq C s^{\alpha-1} .
$$

Note that (I-c) and (I-d) hold for $u_{0}$ which is smooth in its positive and negative phases and is harmonic near the initial free boundary, that is, $-\Delta u_{0}=0$ in the set $\left(\left\{u_{0}>0\right\} \cup\left\{u_{0}<0\right\}\right) \cap\left\{x: \operatorname{dist}\left(x, \partial \Omega_{0}\right) \leq 1\right\}$.

We mention that, roughly speaking, the series of the hypothesis (Ia)-(Id) suggests that we have in mind an initial positive phase $\Omega_{0}$ whose boundary is "almost" $C^{1}$ (that is, a small perturbation of a $C^{1}$ boundary in its Lipschitz norm), and initial data $u_{0}$ whose rescaled profile is "almost" harmonic near $\partial \Omega_{0}$. The smallness assumption on $L$ given in (I-b) is to avoid waiting time phenomena (see [Athanasopoulos et al. 1996; Choi and Kim 2006]), and is most natural in the spirit of previous results [Athanasopoulos et al. 1996; 1998]. The assumption on $u_{0}$ is introduced to ensure that the initial data does not perturb the initial geometry of $\Omega_{0}$ too much (see the discussion in [Choi and Kim 2010]). We expect that regularization of the interface over time should hold for general continuous initial data $u_{0}$.

For a function $u(x, t): \mathbb{R}^{n} \times[0, \infty) \rightarrow \mathbb{R}$, let us write

$$
\Omega(u):=\{u>0\}, \quad \Omega_{t}(u):=\{u(\cdot, t)>0\},
$$


and

$$
\Gamma(u):=\partial\{u>0\}, \quad \Gamma_{t}(u):=\partial\{u(\cdot, t)>0\} .
$$

Since $\Gamma_{0}=\partial\{u(\cdot, 0)>0\}=\partial\{u(\cdot, 0)<0\}$ in our setting, this property is preserved for later times, i.e.,

$$
\Gamma_{t}(u)=\partial\{u(\cdot, t)>0\}=\partial\{u(\cdot, t)<0\} \quad \text { for all } t>0 ;
$$

see [Rogers and Berger 1984; Götz and Zaltzman 1991; Kim and Požár 2011].

For $x_{0} \in \Gamma_{0}=\Gamma_{0}(u)$, we may let $e_{n}=x_{0} /\left|x_{0}\right|$ after a rotation. Then we define

$$
t\left(x_{0}, r\right):=\min \left\{\frac{r^{2}}{u^{+}\left(x_{0}-r e_{n}, 0\right)}, \frac{r^{2}}{u^{-}\left(x_{0}+r e_{n}, 0\right)}\right\} .
$$

Some remarks concerning $t\left(x_{0}, r\right)$ are in order. In one-phase case (where $u^{-} \equiv 0$ ), it was shown in [Choi et al. 2007] that

$$
t\left(x_{0}, r\right) \sim \sup \left\{t>0: u\left(x_{0}+r e_{n}, t\right)=0\right\},
$$

i.e., $t\left(x_{0}, r\right)$ is the time it takes for the free boundary to reach $x_{0}+r e_{n}$. In our (two-phase) case $t\left(x_{0}, r\right)$ is the time it takes for the free boundary to reach $x_{0}+r e_{n}$ if we evolve the free boundary only according to the dominant phase with bigger size of $u$. In particular $\Gamma(u)$ moves at most by distance $r$ by the time $t\left(x_{0}, r\right)$. It turns out that $t\left(x_{0}, r\right)$ is the correct time scale for the solutions in $r$-neighborhood of $x_{0}$ to "mix" and regularize the interface (Theorem 1.1(3)). See the paragraph below Theorem 1.1 for further heuristics based on scaling properties of our problem.

Suppose $u$ is a solution of (ST2) with initial data $u_{0}$ satisfying (Ia)-(Id) with $\Omega_{0}(u) \subset B_{R}(0)$. Due to (Ia)-(Ib), for sufficiently small $r$ and given $x_{0} \in \Gamma_{0}$ the initial free boundary $\Gamma_{0}$ is given by the graph of a Lipschitz function in $B_{r}\left(x_{0}\right)$. After a rotation if necessary, we may assume that

$$
\Omega_{0} \cap B_{r}\left(x_{0}\right)=\left\{x+x_{0}: x=\left(x^{\prime}, x_{n}\right), x_{n} \leq f\left(x^{\prime}\right)\right\},
$$

where $f$ is a Lipschitz function with Lipschitz constant $L<L_{n}$. We summarize our main results:

Theorem 1.1 (cf. Theorem 5.6, Theorem 5.7 and Corollary 5.8). Let $u, \Omega_{0}, r$ and $f$ be as above. There exists $d_{0}>0$ depending only on $n$ and $N_{0}$ such that the following holds for $r \leq d_{0}$ :

(1) In $\Sigma_{r}:=B_{2 r}\left(x_{0}\right) \times\left[t\left(x_{0}, r\right) / 2, t\left(x_{0}, r\right)\right]$, we have

$$
\Gamma(u)=\left\{\left(x+x_{0}, t\right): x=\left(x^{\prime}, x_{n}\right), x_{n} \leq f\left(x^{\prime}, t\right)\right\},
$$

where $f\left(x^{\prime}, t\right)$ is a $C^{1}$ function of space and time. Moreover, there exists a positive dimensional constant $c_{0}$ and $1<m<2$ such that

$$
\begin{aligned}
\left|D_{x^{\prime}} f\left(x^{\prime}, t\right)-D_{x^{\prime}} f\left(y^{\prime}, t\right)\right| & \leq c_{0}\left(-\log \left|\frac{x^{\prime}}{r}-\frac{y^{\prime}}{r}\right|\right)^{-m}, \\
\left|\partial_{t} f\left(x^{\prime}, t\right)-\partial_{t} f\left(x^{\prime}, s\right)\right| & \leq c_{0}\left(-\log \left|\frac{t}{t\left(x_{0}, r\right)}-\frac{s}{t\left(x_{0}, r\right)}\right|\right)^{-1 / 3} .
\end{aligned}
$$

(2) $u$ is a classical solution of (ST2) in $\Sigma_{r}$ in the sense that

(i) Du ${ }^{+}$exists in $\Omega(u)$ and is continuous up to $\bar{\Omega}(u)$; 
(ii) $D u^{-}$exists in $\Omega(u)$ and is continuous up to $\Sigma_{r} \cap\left(\mathbb{R}^{n}-\Omega(u)\right)$;

(iii) the free boundary condition is satisfied in the classical sense, i.e.,

$$
V_{x, t}=\left(\left|D u^{+}\right|-\left|D u^{-}\right|\right)(x, t) \quad \text { on } \Gamma(u) \cap \Sigma_{r} .
$$

(3) There exists a positive dimensional constant $M$ such that

$$
M^{-1} \frac{u^{+}\left(x_{0}-r e_{n}, 0\right)}{r} \leq\left|D u^{+}\right|(x, t) \leq M \frac{u^{+}\left(x_{0}-r e_{n}, 0\right)}{r}
$$

and

$$
M^{-1} \frac{u^{-}\left(x_{0}+r e_{n}, 0\right)}{r} \leq\left|D u^{-}\right|(x, t) \leq M \frac{u^{-}\left(x_{0}+r e_{n}, 0\right)}{r}
$$

in $\Sigma_{r}$.

Remark 1.2. Our result extends to the case where the star-shaped condition (I-a)-(I-b) is replaced by:

(I-ab) $\Omega_{0}$ is locally Lipschitz with a sufficiently small Lipschitz constant.

See the discussion in Section 6.

The one phase version of the above result was proved in [Choi and Kim 2010] (see Theorem 2.16 in Section 2). Let us briefly motivate our result below in the context of the existing literature.

For a given reference point $\left(x_{0}, t_{0}\right) \in \mathbb{R}^{n} \times[0, \infty)$ and positive constants $r$ and $c$, one can rescale the solution $u$ of (ST2) as

$$
\tilde{u}:=\frac{1}{c} u\left(x_{0}+r x, t_{0}+\frac{r^{2}}{c} t\right) .
$$

Then $\tilde{u}$ satisfies the free boundary problem

$$
\begin{cases}r \tilde{u}_{t}-\Delta \tilde{u}=0 & \text { in }\{\tilde{u}>0\} \cup\{\tilde{u}<0\}, \\ V=\left|D \tilde{u}^{+}\right|-\left|D \tilde{u}^{-}\right| & \text {on } \partial\{\tilde{u}>0\},\end{cases}
$$

in a corresponding neighborhood of the origin. Let $e_{1}, \ldots, e_{n}$ be an orthonormal basis of $\mathbb{R}^{n}$, so that $x \in \mathbb{R}^{n}$ can be written as $x=\left(x^{\prime}, x_{n}\right)$, with $x_{n}=x \cdot e_{n}$. Choose $\left(x_{0}, t_{0}\right)=\left(x_{0}, 0\right)$ with $x_{0} \in \Gamma_{0}(u)$. By our hypothesis, after a change of coordinates if necessary, there exists a Lipschitz function $f: \mathbb{R}^{n-1} \rightarrow \mathbb{R}$ with a small Lipschitz constant such that

$$
\Omega_{0}(u) \cap B_{2 r}\left(x_{0}\right)=\left\{x: x_{n} \leq f\left(x^{\prime}\right)\right\} .
$$

Let us choose

$$
c=\max \left\{u^{+}\left(x_{0}-r e_{n}, 0\right), u^{-}\left(x_{0}+r e_{n}, 0\right)\right\}
$$

so that one of $\tilde{u}^{+}\left(-e_{n}, 0\right)$ and $\tilde{u}^{-}\left(+e_{n}, 0\right)$ equals 1 , and the other is less than 1.

Now suppose that we can show the following two conditions:

(A) $|\tilde{u}|(x, t) \leq C$ in $B_{1}(0) \times[0,1]$ with a constant $C>0$ independent of $x_{0}$ and $r$.

(B) The level sets of $\tilde{u}$ are Lipschitz graphs in space and time with small Lipschitz constant in $B_{1}(0) \times[0,1]$. 
Then Theorem 1.1 follows from the results of [Athanasopoulos et al. 1996] applied to $\tilde{u}$. Indeed, (B) can be replaced by a relaxed version $\left(\mathrm{B}^{\prime}\right)$ as stated below, which is sufficient to derive Theorem 1.1 due to the results of [Athanasopoulos et al. 1998].

$\left(\mathrm{B}^{\prime}\right)$ The level sets of $\tilde{u}$ are $\epsilon$-monotone with respect to cones of directions $W_{x}\left(\theta^{x}, e\right)$ and $W_{t}\left(\theta^{t}, v\right)$ with $v \in \operatorname{span}\left(e_{n}, e_{t}\right)$, and $\pi / 2-\theta^{x}$ and $\epsilon$ sufficiently small.

(For the meaning of $\epsilon$-monotonicity and the space and time cones $W_{x}$ and $W_{t}$, see Definition 2.1.)

In our case $(\mathrm{A})$ can be verified using previously known results in the one-phase Stefan problem (Lemma 3.3 and Lemma 3.4). Unfortunately, as shown in [Choi and Kim 2010], verifying (B') for all scales $r$ turns out to be as difficult as showing (B) or the full regularity of $u$. Since $\tilde{u}$ no longer satisfies the heat equation, one loses control of the change of $u$ over time. For this reason it is necessary to show $\left(\mathrm{B}^{\prime}\right)$ for all level sets of $\tilde{u}$, not just for the free boundary $\Gamma(\tilde{u})$. Indeed, in this article we will first show that $\tilde{u}$ (scaled correspondingly for the two-phase) is $\epsilon$-monotone in the space variable (Lemma 3.1), and then we show that $\Gamma(\tilde{u})$ is $\epsilon$-monotone in the space-time variables (Corollary 4.4 and Lemma 4.7). Then in Section 5 we use the $\epsilon$-monotonicity obtained from previous sections, the almost-harmonicity of $\tilde{u}$ (Lemma 3.6), as well as the iteration methods originating from [Athanasopoulos et al. 1996; 1998] to show directly that $\tilde{u}$ is a classical solution and $u$ satisfies (B) and ( $\mathrm{B}^{\prime}$ ) (Section 5). The arguments in Section 5 are mostly drawn from [Athanasopoulos et al. 1996; 1998] as well as [Choi et al. 2007; 2009].

Let us now illustrate the underlying ideas in the analysis in Section 4, where we show the $\epsilon$-monotonicity of the solution over time. In terms of the original solution $u$, verifying $(\mathrm{A})$ and $\left(\mathrm{B}^{\prime}\right)$ corresponds to analyzing $u$ over the time interval $\left[0, t\left(x_{0}, r\right)\right]$, where $t\left(x_{0}, r\right)$ is given by

$$
t\left(x_{0}, r\right):=r^{2} / c
$$

and $c$ is as given in (1-5). Note that $t\left(x_{0}, r\right)$ coincides with the one given by (1-3).

Heuristically speaking, there are two possible scenarios for interface regularization, depending on its initial configuration in the local neighborhood:

(1) One of the phases has much bigger flux than the other, i.e.,

$$
u^{+}\left(x_{0}-s e_{n}, 0\right) \gg u^{-}\left(x_{0}+s e_{n}, 0\right) \quad \text { or } \quad u^{-}\left(x_{0}-s e_{n}, 0\right) \ll u^{-}\left(x_{0}+s e_{n}, 0\right)
$$

for $s$ comparable to $r$.

In this case one-phase-like phenomena (regularization by the dominant phase as obtained in Theorem 2.16) are expected. As mentioned above, in this case the time interval for regularization of the free boundary in $r$-neighborhood is proportional to the distance it has traveled.

(2) Both phases are in balance, i.e.,

$$
u^{+}\left(x_{0}-s e_{n}, 0\right) \sim u^{-}\left(x_{0}+s e_{n}, 0\right)
$$

for $s$ comparable to $r$. 
In this case one expects regularization due to competition between two phases, resulting in Lipschitz-like behavior over time. Again the corresponding time interval for regularization amounts to $t\left(x_{0}, r\right)$ given in (1-3).

To make the above heuristics rigorous, in Section 4 we will introduce a decomposition procedure based on Harnack-type inequalities, which illustrates local dynamics near the free boundary: roughly speaking, for a given $r>0$ we divide $B_{r}\left(x_{0}\right) \times\{t=0\}$ into regions where (1-6) holds for $0<s \ll r$ (balanced region) and the rest of domain (unbalanced region). (See detailed definitions of these regions in Section 4.) Of course the main issue is whether the dynamics of one region affect the other, in particular whether the one-phase-type dynamics of the unbalanced region breaks the property (1-6) in the balanced region for future times. We will show that this does not happen (Proposition 4.3), due to a fast regularization property in the unbalanced region (Proposition 3.7 and Lemma 4.7) as well as Harnack-type inequalities (Lemmas 4.5 and 4.6) in the balanced region.

Let us finish this section with an outline of the paper. In Section 2 we introduce preliminary results and notation, including the regularity results in the one-phase Stefan problem (Theorem 2.16). Sections 3 to 5 consist of the proof of Theorem 1.1; in Section 3 we prove some properties on the evolution of solutions of (ST2) with star-shaped data. In addition to Harnack inequalities, we show that the solution stays near the star-shaped profile for a unit time (Lemma 3.1), which in turn yields that the solution stays very close to harmonic functions (Lemma 3.6). This establishes that $\left(\mathrm{B}^{\prime}\right)$ holds in the space variable. Making use of the results in Section 3, we perform a decomposition procedure in Section 4, to show that (A) holds for $\tilde{u}$ (Proposition 4.3) and that $\left(\mathrm{B}^{\prime}\right)$ holds for $\Gamma(\tilde{u})$ (Corollary 4.4). This completes the main step in our analysis. In Section 5 we describe the rather technical iteration procedure leading to further regularization, and we complete the proof of Theorem 1.1 by combining arguments from [Athanasopoulos et al. 1996; 1998; Choi et al. 2007; 2009] (Theorem 5.7 and Corollary 5.8). In Section 6 we discuss a generalized proof of the corresponding regularization result (Theorem 6.1) when the star-shapedness of the initial data (I-a) and (I-b) are replaced by the local version (I-ab).

\section{Preliminary lemmas and notation}

We introduce some notation.

- For $x \in \mathbb{R}^{n}$, denote by $x=\left(x^{\prime}, x_{n}\right) \in \mathbb{R}^{n-1} \times \mathbb{R}$, where $x_{n}=x \cdot e_{n}$.

- Let $B_{r}(x)$ be the space ball of radius $r$, centered at $x$.

- Let $Q_{r}:=B_{r}(0) \times\left[-r^{2}, r^{2}\right]$ be the parabolic cube and let $K_{r}:=B_{r}(0) \times[-r, r]$ be the hyperbolic cube.

- A caloric function in $\Omega \cap Q_{r}$ will denote a nonnegative solution of the heat equation, vanishing along the lateral boundary of $\Omega$.

- For $x_{0} \in \Gamma_{0}$ and $e_{n}=x_{0} /\left|x_{0}\right|$, define

$$
t\left(x_{0}, d\right):=\min \left\{\frac{d^{2}}{u^{+}\left(x_{0}-d e_{n}, 0\right)}, \frac{d^{2}}{u^{-}\left(x_{0}+d e_{n}, 0\right)}\right\} .
$$


- $C$ is called a universal constant if it depends only on the dimension $n$ and the regularity constant $N_{0}$ of $u_{0}$.

- We say $a \sim b$ if there exists a dimensional constant $C>0$ such that $C^{-1} b \leq a \leq C b$.

- Lastly let us recall the definition of $\epsilon$-monotonicity. Let $W_{x}\left(\theta^{x}, e\right)$ and $W_{t}\left(\theta^{t}, v\right)$ with $e \in \mathbb{R}^{n}$ and $v \in \operatorname{span}\left(e_{n}, e_{t}\right)$, respectively, denote a spatial circular cone of aperture $2 \theta^{x}$ and axis in the direction of $e$, and a two-dimensional space-time cone in $\left(e_{n}, e_{t}\right)$ plane of aperture $2 \theta^{t}$ and axis in the direction of $\nu$.

Definition 2.1. (a) Given $\epsilon>0$, a function $w$ is called $\epsilon$-monotone in the direction $\tau$ if

$$
u(p+\lambda \tau) \geq u(p) \text { for any } \lambda \geq \epsilon .
$$

(b) $w$ is $\epsilon$-monotone in a cone of directions $W_{x}\left(\theta^{x}, e\right)$ or $W_{t}\left(\theta^{t}, \nu\right)$ if $w$ is $\epsilon$-monotone in every direction in the cone.

Next we state preliminary results that are important in our analysis. The first lemma is a direct consequence of the interior Harnack inequalities proved in [Caffarelli and Cabré 1995].

Lemma 2.2. Suppose $w(x): \mathbb{R}^{n} \rightarrow \mathbb{R}$ has bounded Laplacian. Then $w$ is Hölder continuous with its constant depending on the Laplacian bound.

Lemma 2.3 [Fabes et al. 1984, Theorem 3]. Let $\Omega$ be a domain in $\mathbb{R}^{n} \times \mathbb{R}$ such that $(0,0)$ is on its lateral boundary. Suppose $\Omega$ is a $\mathrm{Lip}^{1,1 / 2}$ domain, i.e.,

$$
\Omega=\left\{\left(x^{\prime}, x_{n}, t\right):\left|x^{\prime}\right|<1,\left|x_{n}\right|<2 L,|t|<1, x_{n} \leq f\left(x^{\prime}, t\right)\right\},
$$

where $f$ satisfies $\left|f\left(x^{\prime}, t\right)-f\left(y^{\prime}, s\right)\right| \leq L\left(\left|x^{\prime}-y^{\prime}\right|+|t-s|^{1 / 2}\right.$.) If $u$ is a caloric function in $\Omega$, then there exists $C=C(n, L)$, where $L$ is the Lipschitz constant for $\Omega$, such that

$$
\frac{u(x, t)}{v(x, t)} \leq C \frac{u\left(-L e_{n}, \frac{1}{2}\right)}{v\left(-L e_{n},-\frac{1}{2}\right)}
$$

for $(x, t) \in Q_{1 / 2}$.

Lemma 2.4 [Athanasopoulos et al. 1996, Theorem 1]. Let $\Omega$ be a Lipschitz domain in $\mathbb{R}^{n} \times \mathbb{R}$, i.e.,

$$
Q_{1} \cap \Omega=Q_{1} \cap\left\{(x, t): x_{n} \leq f\left(x^{\prime}, t\right)\right\},
$$

where $f$ satisfies $|f(x, t)-f(y, s)| \leq L(|x-y|+|t-s|)$. Let $u$ be a caloric function in $Q_{1} \cap \Omega$ with $(0,0) \in \partial \Omega$ and $u\left(-e_{n}, 0\right)=m>0$ and $\sup _{Q_{1}} u=M$. Then there exists a constant $C$, depending only on $n, L, m / M$ such that

$$
u\left(x, t+\rho^{2}\right) \leq C u\left(x, t-\rho^{2}\right)
$$

for all $(x, t) \in Q_{1 / 2} \cap \Omega$ and for $0 \leq \rho \leq d_{x, t}$.

Lemma 2.5 [Athanasopoulos et al. 1996, Lemma 5]. Let $u$ and $\Omega$ be as in Lemma 2.4. Then there exist $a, \delta>0$ depending only on $n, L, m / M$ such that

$$
w_{+}:=u+u^{1+a} \quad \text { and } \quad w_{-}:=u-u^{1+a}
$$


are subharmonic and superharmonic, respectively, in $Q_{\delta} \cap \Omega \cap\{t=0\}$.

Next we state several properties of harmonic functions:

Lemma 2.6 [Dahlberg 1979]. Let $u_{1}, u_{2}$ be two nonnegative harmonic functions in a domain $D$ of $\mathbb{R}^{n}$ of the form

$$
D=\left\{\left(x^{\prime}, x_{n}\right) \in \mathbb{R}^{n-1} \times \mathbb{R}:\left|x^{\prime}\right|<2,\left|x_{n}\right|<2 L, x_{n}>f\left(x^{\prime}\right)\right\},
$$

with $f$ a Lipschitz function with constant less than $L$ and $f(0)=0$. Assume further that $u_{1}=u_{2}=0$ along the graph of $f$. Then in

$$
D_{1 / 2}=\left\{\left|x^{\prime}\right|<1,\left|x_{n}\right|<L, x_{n}>f\left(x^{\prime}\right)\right\}
$$

we have

$$
0<C_{1} \leq \frac{u_{1}\left(x^{\prime}, x_{n}\right)}{u_{2}\left(x^{\prime}, x_{n}\right)} \cdot \frac{u_{2}(0, L)}{u_{1}(0, L)} \leq C_{2},
$$

with $C_{1}, C_{2}$ depending only on $L$.

Lemma 2.7 [Jerison and Kenig 1982]. Let D, $u_{1}$ and $u_{2}$ be as in Lemma 2.6. Assume further that

$$
\frac{u_{1}(0, L / 2)}{u_{2}(0, L / 2)}=1 \text {. }
$$

Then, $u_{1}\left(x^{\prime}, x_{n}\right) / u_{2}\left(x^{\prime}, x_{n}\right)$ is Hölder continuous in $\bar{D}_{1 / 2}$ for some coefficient $\alpha$, both $\alpha$ and the $C^{\alpha}$ norm of $u_{1} / u_{2}$ depending only on $L$.

Lemma 2.8 [Caffarelli 1987]. Let $u$ be as in Lemma 2.6. Then there exists $c>0$ depending only on $L$ such that for $0<d<c, \frac{\partial}{\partial x_{n}} u(0, d) \geq 0$ and

$$
C_{1} \frac{u(0, d)}{d} \leq \frac{\partial u}{\partial x_{n}}(0, d) \leq C_{2} \frac{u(0, d)}{d},
$$

where $C_{i}=C_{i}(M)$.

Lemma 2.9 [Jerison and Kenig 1982, Lemma 4.1]. Let $\Omega$ be Lipschitz domain contained in $B_{10}(0)$. There exists a dimensional constant $\beta_{n}>0$ such that for any $\zeta \in \partial \Omega, 0<2 r<1$ and positive harmonic function $u$ in $\Omega \cap B_{2 r}(\zeta)$, if u vanishes continuously on $B_{2 r}(\zeta) \cap \partial \Omega$, then for $x \in \Omega \cap B_{r}(\zeta)$,

$$
u(x) \leq C\left(\frac{|x-\zeta|}{r}\right)^{\beta_{n}} \sup \left\{u(y): y \in \partial B_{2 r}(\zeta) \cap \Omega\right\},
$$

where $C$ depends only on the Lipschitz constants of $\Omega$.

Next, we point out that we use the notion of viscosity solutions for our investigation. When $\left\{u_{0}=0\right\}$ is of zero Lebesgue measure, it was proved in [Kim and Požár 2011] that the viscosity solution of (ST2) is unique and coincides with the usual weak solutions. (See [Kim and Požár 2011] for the definition as well 
as other properties of viscosity solutions.) Below we state important properties of viscosity solutions for (ST2) that relate our solutions to the one-phase version of our problem,

$$
\begin{cases}u_{t}-\Delta u=0 & \text { in }\{u>0\} \\ u_{t} /|D u|=|D u| & \text { on } \partial\{u>0\}, \\ u(\cdot, 0)=u_{0} \geq 0 & \end{cases}
$$

Lemma 2.10. Suppose $u$ is a viscosity solution of (ST2). Then:

(a) $u$ is caloric in its positive and negative phases.

(b) $-u$ is also a viscosity solution of (ST2) with boundary data $-g$.

(c) $u^{+}=\max (u, 0)\left(\right.$ or $\left.u^{-}=-\min (u, 0)\right)$ is a viscosity subsolution of (ST1) with initial data $u_{0}^{+}\left(\right.$or $\left.u_{0}^{-}\right)$.

We say that a pair of functions $u_{0}, v_{0}: \bar{D} \rightarrow[0, \infty)$ are (strictly) separated (denoted by $u_{0} \prec v_{0}$ ) in $D \subset \mathbb{R}^{n}$ if:

(i) The support of $u_{0}, \operatorname{supp}\left(u_{0}\right)=\overline{\left\{u_{0}>0\right\}}$, restricted to $\bar{D}$ is compact.

(ii) $u_{0}(x)<v_{0}(x)$ in $\operatorname{supp}\left(u_{0}\right) \cap \bar{D}$.

Lemma 2.11 [Kim and Požár 2011, Comparison principle]. Let $u, v$ be, respectively, viscosity sub- and supersolutions of (ST2) in $D \times(0, T) \subset Q$ with initial data $u_{0} \prec v_{0}$ in $D$. If $u \leq v$ on $\partial D$ and $u<v$ on $\partial D \cap \bar{\Omega}(u)$ for $0 \leq t<T$, then $u(\cdot, t) \prec v(\cdot, t)$ in $D$ for $t \in[0, T)$.

Below we state a distance estimate for the free boundary and Harnack inequality for the one-phase solution $u$ of (ST1).

Lemma 2.12 [Choi and Kim 2010, Lemma 2.2]. Let $u$ be given as in Theorem 2.16. There exists $t_{0}=t_{0}\left(N_{0}, M_{0}, n\right)>0$ such that if $x_{0} \in \Gamma_{0}$ and $t \leq t_{0}$, then

$$
\frac{1}{C} t^{1 /(2-\alpha)} \leq d\left(x_{0}, t\right) \leq C t^{1 /(2-\beta)},
$$

where $\alpha$ and $\beta$ are given in (1-2), $C$ depends on $N_{0}, M_{0}$ and $n$, and $d\left(x_{0}, t\right)$ denotes the distance that $\Gamma$ moved from the point $x_{0}$ during the time $t$, i.e.,

$$
d\left(x_{0}, t\right):=\sup \left\{d: u\left(x_{0}+d e_{n}, t\right)>0\right\} .
$$

Lemma 2.13 [Choi and Kim 2010, Lemma 2.3]. Let $u$ be given as in Theorem 2.16. There exists $d_{0}$ depending on $N_{0}, M_{0}$ and $n$ such that if $x_{0} \in \Gamma_{0}$ and $d \leq d_{0}$, then

$$
u\left(x_{0}-d e_{n}, t\right) \leq C u\left(x_{0}-d e_{n}, 0\right) \quad \text { for } 0 \leq t \leq t\left(x_{0}, d\right),
$$

where $C$ depends on $N_{0}, M_{0}$ and $n$.

The following monotonicity formula by Alt-Caffarelli-Friedman prevents the scenario that both phases compete with large pressure in our problem. 
Lemma 2.14 [Alt et al. 1984]. Let $h_{+}$and $h_{-}$be nonnegative continuous functions in $B_{1}(0)$ such that $\Delta h_{ \pm} \geq 0$ and $h_{+} \cdot h_{-}=0$ in $B_{1}(0)$. Then the functional

$$
\phi(r)=\frac{1}{r^{4}} \int_{B_{r}(0)} \frac{\left|D h_{+}\right|^{2}}{|x|^{n-2}} d x \int_{B_{r}(0)} \frac{\left|D h_{-}\right|^{2}}{|x|^{n-2}} d x
$$

is monotone increasing in $r, 0<r<1$.

Corollary 2.15. Let $\partial \Omega_{0} \subset \mathbb{R}^{n}$ be star-shaped with respect to the ball $B_{1}(0) \subset \Omega_{0}$ and suppose that $B_{4 / 3}(0) \subset \Omega_{0} \subset B_{5 / 3}(0)$. Let $h_{+}$be the harmonic function in $\Omega_{0}-B_{1}(0)$ with boundary values $h_{+}=0$ on $\partial \Omega_{0}$, and $h_{+}=1$ on $\partial B_{1}(0)$. Let $h_{-}$be the harmonic function in $B_{2}(0)-\Omega_{0}$ with boundary values $h_{-}=0$ on $\partial \Omega_{0}$, and $h_{-}=1$ on $\partial B_{2}(0)$. Then there exists a sufficiently large dimensional constant $M>0$ such that

$$
\frac{h_{+}\left(x_{0}-r e_{n}\right)}{r} \geq M \text { implies } \frac{h_{-}\left(x_{0}+r e_{n}\right)}{r} \leq 1
$$

for $x_{0} \in \partial \Omega_{0}, e_{n}=x /|x|$ and $0 \leq r \leq 1 / 6$.

Proof. This follows from Lemma 2.14, since

$$
\begin{aligned}
\left(\frac{h_{+}\left(x_{0}-r e_{n}\right)}{r} \cdot \frac{h_{-}\left(x_{0}+r e_{n}\right)}{r}\right)^{2} & \sim \frac{1}{(2 r)^{4}} \int_{B_{r / 2}\left(x_{0}-r e_{n}\right)} \frac{\left|D h_{+}\right|^{2}}{\left|x-x_{0}\right|^{n-2}} d x \cdot \int_{B_{r / 2}\left(x_{0}+r e_{n}\right)} \frac{\left|D h_{-}\right|^{2}}{\left|x-x_{0}\right|^{n-2}} d x \\
& \leq \frac{1}{(2 r)^{4}} \int_{B_{2 r}\left(x_{0}\right)} \frac{\left|D h_{+}\right|^{2}}{\left|x-x_{0}\right|^{n-2}} d x \cdot \int_{B_{2 r}\left(x_{0}\right)} \frac{\left|D h_{-}\right|^{2}}{\left|x-x_{0}\right|^{n-2}} d x \\
& =\phi(2 r) \leq \phi(1 / 3) \leq C_{n} .
\end{aligned}
$$

Lastly, let us finish this section by stating the results obtained in [Choi and Kim 2010] for the one-phase version of our problem in the local setting:

Theorem 2.16 [Choi and Kim 2010, Theorem 0.1]. Suppose a nonnegative function $u(x, t)$ is a solution of (ST1) in $B_{2}(0) \times[0,1], 0 \in \Gamma_{0}(u)$, with the initial data $u_{0} \geq 0$ satisfying $(I-b),(I-c)$ and $(I-d)$ in $B_{2}(0)$. Suppose the initial data satisfies

$$
\{u(x, 0) \geq 0\}=\left\{x+x_{0}: x_{n} \leq f\left(x^{\prime}\right)\right\}
$$

in $B_{1}(0)$, where $f$ is a Lipschitz function with Lipschitz constant $L<L_{n}$. Further, suppose $u_{0}\left(-e_{n}\right)=1$ and $\sup _{B_{2}(0) \times[0,1]} u \leq M_{0}$.

For given $r>0$, let us define

$$
t\left(x_{0}, r\right):=\frac{r^{2}}{u\left(x_{0}+r e_{n}, 0\right)} .
$$

Then there exists a small $c_{0}>0$ depending on $M_{0}$ and $n$ such that in $\Sigma_{r}=B_{r}\left(x_{0}\right) \times\left[t\left(x_{0}, r\right) / 2, t\left(x_{0}, r\right)\right]$ for $r \leq c_{0}$, we have:

(1) Theorem 1.1(1) holds for $u$. 
(2) $u$ is a classical solution of (ST1) in $\Sigma_{r}$ in the sense that the spatial derivative Du exists in $\Omega(u)$ and is continuous up to $\bar{\Omega}(u)$, and the free boundary condition is satisfied in the classical sense, i.e.,

$$
V_{x, t}=|D u|(x, t) \quad \text { on } \Gamma(u) \cap \Sigma_{r} .
$$

(3) There exists a positive constant $M$ depending on $M_{0}$ and $n$ such that

$$
M^{-1} \frac{u\left(x_{0}-r e_{n}, 0\right)}{r} \leq|D u|(x, t) \leq M \frac{u\left(x_{0}-r e_{n}, 0\right)}{r} .
$$

(4) If $x \in \Gamma_{0}(u) \cap B_{c_{0}}(0)$ and $x+r e_{n} \in \Gamma_{t}(u) \cap B_{c_{0}}(0)$, then

$$
M^{-1} \frac{u\left(x-r e_{n}, 0\right)}{r} \leq\left|D u\left(x+r e_{n}, t\right)\right|=V_{x+r e_{n}, t} \leq M \frac{u\left(x-r e_{n}, 0\right)}{r},
$$

where $M$ depends on $n$ and $M_{0}$. In particular,

$$
\frac{r}{t} \sim\left|D u\left(x+r e_{n}, t\right)\right| \sim \frac{u\left(x-r e_{n}, 0\right)}{r} .
$$

Theorem 2.16 states that the free boundary regularizes in a scale proportional to the distance it has traveled. Note that the regularity results hold up to the initial time and all the regularity assumptions are imposed only on the initial data.

\section{Properties of solutions with star-shaped initial data}

Lemma 3.1. If $\Omega_{0}$ and $u_{0}$ are star-shaped with respect to the ball $B_{r_{0}}(0) \subset \Omega_{0}$, then $\Omega_{t}(u)$ and $u(\cdot, t)$ stays $\sigma$-close to star-shaped for all $0 \leq t \leq \frac{1}{3} \sigma^{1 / 5}$ (see Figure 2).

Proof. Step 1. For any $a>0$, the parabolic scaling $(x, t) \rightarrow\left(a x, a^{2} t\right)$ preserves both the heat operator

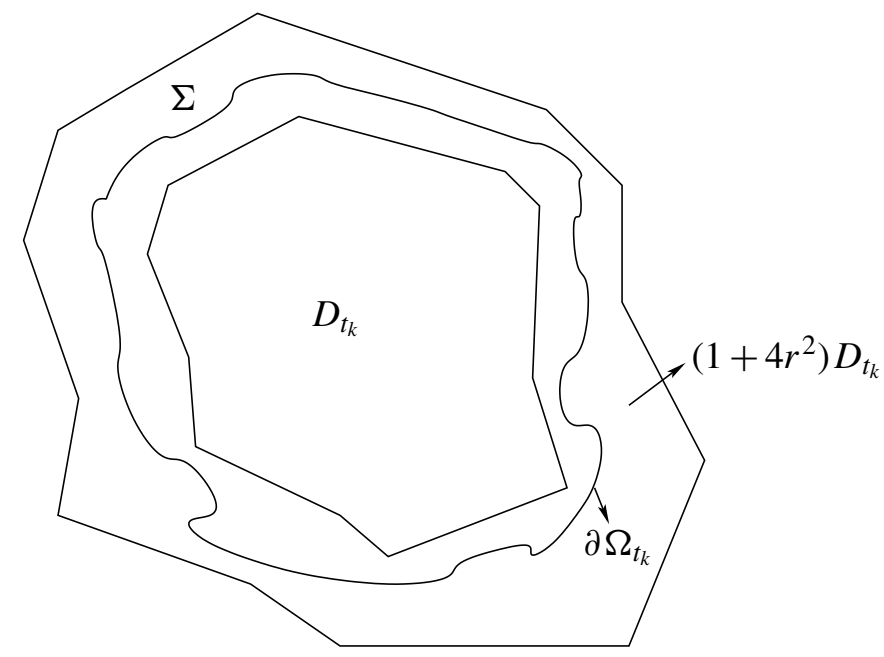

Figure 2. Approximation of the positive phase by a star-shaped domain. 
and the boundary motion law in (ST2). Therefore, for any $\sigma>0$ the function

$$
u_{1}(x, t):=u\left((1+\sigma)\left(x-x_{0}\right)+x_{0},(1+\sigma)^{2} t\right)
$$

is also a viscosity solution of (ST2) with corresponding initial data.

Step 2. Choose $x_{0} \in B_{r_{0}}(0)$. Take a small $c_{0}>0$ such that $B_{r_{0}+c_{0}}(0) \subset \Omega_{0}$. We claim that for $0 \leq \delta \leq \sigma^{6 / 5}$,

$$
u_{1}(x, 0) \leq u(x, \delta) \quad \text { in } B_{R}(0)-B_{r_{0}+c_{0}}(0)
$$

if $\sigma$ is small enough. To show (3-1), let us introduce another function

$$
\tilde{u}(x, 0):=u\left(\left(1+\frac{1}{2} \sigma\right)\left(x-x_{0}\right)+x_{0}, 0\right) .
$$

Also let $v^{*}$ be the solution of the one phase problem (ST1) with initial data $u_{0}^{-}$, and with $v^{*}=1$ on $\partial B_{R}(0)$. Note also that, due to Lemma 2.10, $u^{-}$is a subsolution of (ST1) with initial data $v^{*}(x, 0)=u^{-}(x, 0)$.

Thus by Lemma 2.11, $v^{*} \leq u^{-}$. It follows that $\Omega_{t}\left(v^{*}\right) \subset \Omega_{t}(u) \subset \Omega_{t}(u)$. Hence by Lemma 2.12 applied to $-v^{*}$,

$$
\Omega_{0}(\tilde{u}) \subset \Omega_{t}(u) \quad \text { for } 0 \leq t \leq \sigma^{7 / 6}
$$

Moreover, due to our assumption,

$$
\tilde{u}(x, 0) \leq u_{0}(x)
$$

Therefore, the maximum principle for caloric functions implies

$$
w(x, t) \leq u(x, t)
$$

where $w$ solves the heat equation in the cylindrical domain $D=\Omega_{0}(\tilde{u}) \times\left[0, \sigma^{7 / 6}\right]$ with initial data $\tilde{u}(x, 0)$ and zero boundary data on $\partial \Omega_{0}(\tilde{u}) \times\left[0, \sigma^{7 / 6}\right]$.

Now $w_{t}$ solves the heat equation in $D$,

$$
w_{t}=\Delta w \geq-C \text { at } t=0 \text { and } w_{t}=0 \text { on } \partial \Omega_{0}(\tilde{u}) .
$$

Therefore we conclude that $w_{t} \geq-C$ in $D$. In particular

$$
w(x, \delta) \geq \tilde{u}(x, 0)-C \delta .
$$

Next we compare $u_{1}(x, 0)$ with $w(x, \delta)$. Observe that for $x \in B_{R}(0)-B_{r_{0}+c_{0}}(0)$,

$$
\begin{aligned}
u_{1}(x, 0)=\tilde{u}(x, 0)+\int_{\sigma / 2}^{\sigma}\left(\left(x-x_{0}\right) \cdot D u\left((1+s)\left(x-x_{0}\right)+x_{0}, 0\right)\right) d s & \leq \tilde{u}(x, 0)-c_{0} \sigma^{7 / 6} \\
& \leq \tilde{u}(x, 0)-C \sigma^{6 / 5} \\
& \leq w(x, \delta) \leq u(x, \delta)
\end{aligned}
$$

for $0 \leq \delta \leq \sigma^{6 / 5}$, where the first inequality follows from our assumption (I-d) on $u_{0}$, the second inequality follows if $\sigma$ is sufficiently small, and the third inequality follows from (3-2). Hence we conclude (3-1). Step 3. Our goal is to prove that for $0 \leq \delta \leq \sigma^{6 / 5}$,

$$
u_{1}(x, t) \leq u_{2}(x, t):=u(x, t+\delta)
$$


in $\left(B_{R}(0)-B_{r_{0}+c_{0}}(0)\right) \times\left[0, \sigma^{1 / 5}\right]$. Note that the inequality holds at $t=0$ by Step 2 . However, we need a few more arguments since we do not know yet if the lateral boundary data on $\partial B_{r_{0}+c_{0}}(0)$ is properly ordered.

Suppose

$$
\Omega\left(u_{1}\right) \subset \Omega(u) \text { for } 0 \leq t \leq t_{0}
$$

and $\Omega\left(u_{1}\right)$ contacts $\partial \Omega(u)$ for the first time at $t=t_{0}$. Observe then that

$$
f(x, t):=u(x, t+\delta)-u_{1}(x, t)
$$

solves the heat equation in $\Omega\left(u_{1}\right)$ with nonnegative boundary data for $0 \leq t \leq t_{0}$, with

$$
f(x, 0) \geq 0 \quad \text { in } B_{R}(0)-B_{r_{0}+c_{0}}(0) .
$$

Indeed, following the computation given above, it follows that

$$
f(x, 0) \geq c_{0} \sigma \quad \text { in } B_{r_{0}+c_{0}}(0)-B_{r_{0}+c_{0} / 2}(0) .
$$

On the other hand, due to the fact that $w_{t} \geq-C$ and $\delta \leq \sigma^{6 / 5}$, we have

$$
f(x, 0) \geq(w(x, \delta)-w(x, 0))+\left(w(x, 0)-u_{1}(x, 0)\right) \geq-C \sigma^{6 / 5} \quad \text { in } B_{r_{0}+c_{0} / 2}(0) .
$$

Therefore we have

$$
f(x, t)>0 \quad \text { on } \partial B_{r_{0}+c_{0}}(0) \times\left[0, t_{0}\right]
$$

if $t_{0} \ll 1$. But then this contradicts Lemma 2.11 applied to the region $\left(B_{R}(0)-B_{r_{0}+c_{0}}(0)\right) \times\left[0, t_{0}\right]$.

Step 4. From (3-3) of Step 3, we obtain

$$
u\left((1+\sigma)\left(x-x_{0}\right)+x_{0},(1+\sigma)^{2} t\right) \leq u(x, t+\delta)
$$

in $\left(B_{R}(0)-B_{r_{0}+c_{0}}(0)\right) \times\left[0, \sigma^{1 / 5}\right]$ for any $x_{0} \in B_{r_{0}}(0)$, as long as $\sigma$ and $\delta$ are sufficiently small and satisfy $0 \leq \delta \leq \sigma^{6 / 5}$. As a result, for $0 \leq t \leq \frac{1}{3} \sigma^{1 / 5}$, we can choose $\delta=\sigma(2+\sigma) t \leq \sigma^{6 / 5}$ such that

$$
(1+\sigma)^{2} t=t+\delta
$$

It follows then from (3-4) that the function $u(\cdot, t)$ is $\sigma$-monotone with respect to the cone of directions $W_{x}$ in $\left(B_{R}(0)-B_{r_{0}+c_{0}}(0)\right)$ for $t \in\left[0, \frac{1}{3} \sigma^{1 / 5}\right]$. (Here $W_{x}=\left\{v \in S^{n}: v=\left(x-x_{0}\right) /\left|x-x_{0}\right|\right.$ for some $\left.x_{0} \in B_{r_{0}}(0)\right\}$.)

Remark 3.2. For $x \in \Gamma_{0}$, we may let $e_{n}=x /|x|$ after a rotation. Then, due to (I-b),

$$
t(x, d):=\min \left\{\frac{r^{2}}{u^{+}\left(x-r e_{n}, 0\right)}, \frac{r^{2}}{u^{-}\left(x+r e_{n}, 0\right)}\right\} \in\left[r^{7 / 6}, r^{5 / 6}\right] \ll r^{4 / 5},
$$

where $t(x, r)$ is the time it takes for the free boundary to regularize in $B_{r}(0)$. Therefore, we have, for $0 \leq t \leq t\left(x_{0}, r\right)$,

$$
u(\cdot, t) \text { is } r^{4} \text {-monotone with respect to } W_{x} \text { in }\left(B_{R}(0)-B_{r_{0}+c_{0}}(0)\right) .
$$


This property will ensure that our solution $u$ has its level sets close to Lipschitz graphs in the space variable in an appropriate scale, which serves as the first step towards the regularization argument; see Lemma 3.6.

Lemma 3.3 (Harnack at $t=0$ ). Let $u$ be as in Theorem 1.1. For $x \in \Gamma_{0}$, we may set $e_{n}=x /|x|$ after a rotation. Then for all $s>0$ and for $0 \leq t \leq t(x, s)$ we have

$$
u^{+}\left(x-s e_{n}, t\right) \leq C_{1} u^{+}\left(x-s e_{n}, 0\right) \quad \text { and } u^{-}\left(x+s e_{n}, t\right) \leq C_{1} u^{-}\left(x+s e_{n}, 0\right),
$$

where $e_{n}=x /|x|$.

Proof. Let $v^{* *}$ solve the one-phase Stefan problem (ST1) with initial data $v_{0}^{* *}(x)=u_{0}^{+}(x)$. Then $v^{* *}$ is also a solution of (ST2) with $u_{0}(x) \leq v_{0}^{* *}(x)$, and thus by Lemma 2.11 we have

$$
u(x, t) \leq v^{* *}(x, t) .
$$

Therefore it follows from one-phase Harnack inequality applied for $v^{* *}(x, t)$ that

$$
u^{+}\left(x-s e_{n}, t\right) \leq v^{* *}\left(x-s e_{n}, t\right) \leq C_{1} v^{* *}\left(x-s e_{n}, 0\right)=C_{1} u\left(x-s e_{n}, 0\right)
$$

for $0 \leq t \leq t_{0}$, where $t_{0}=s^{2} / u\left(x-s e_{n}, 0\right) \geq t(x, s)$.

As for $u^{-}(x, t)$, we compare $u^{-}$with the solution $v^{*}$ of (ST1) with initial data $v_{0}^{*}(x)=u_{0}^{-}(x)$ and with boundary data $v^{*}=1$ on $\partial B_{R}(0)$. The rest of the argument is parallel to the above one.

Lemma 3.4 (backward Harnack at $t=0$ ). Let $u$ be as in Theorem 1.1. Let $x \in \Gamma_{0}$ and let $e_{n}=x /|x|$ after a rotation. Then for $s>0$ and for $0 \leq t \leq t(x, s)$,

$$
u^{+}\left(x-s e_{n}, 0\right) \leq C_{1} u^{+}\left(x-s e_{n}, t\right) \quad \text { and } \quad u^{-}\left(x+s e_{n}, 0\right) \leq C_{1} u^{-}\left(x+s e_{n}, t\right) .
$$

Proof. We will only show the lemma for $u^{+}$. The other part follows by a parallel argument. Let $v^{*}$ solve the one phase problem (ST1) with initial data $u_{0}^{-}$and with boundary data 1 on $\partial B_{R}(0)$. Then $-v^{*}$ is also a solution of (ST2) with $-v_{0}^{*} \leq u_{0}$, and thus by Lemma $2.11,-v^{*} \leq u$. This inequality implies that

$$
\left\{v^{*}=0\right\} \subset\{u \geq 0\} .
$$

Note that $\Omega\left(v^{*}\right)$ moves according to the one-phase dynamics, which have been studied in detail in [Choi and Kim 2006]. In particular we know that $\Omega\left(v^{*}\right)$ will be Lipschitz at each time. Moreover, for a boundary point $(x, t) \in \Gamma\left(v^{*}\right)$ and $d:=\operatorname{dist}\left(x, \Gamma_{0}\left(v^{*}\right)\right)$, the normal velocity $V_{x, t}$ satisfies

$$
V_{x, t}=\left|D v^{*}(x, t)\right| \sim \frac{v^{*}\left(x+2 d e_{n}, 0\right)}{2 d} \leq d^{\beta-1} \leq t^{(\beta-1) /(2-\alpha)},
$$

where the last inequality follows from Lemma 2.12. Let $v_{*}(x, t)$ solve the heat equation in $\left\{v^{*}=0\right\}$ with initial data $u_{0}(x)$ and boundary data 0 on the lateral boundary of $\partial\left\{v^{*}=0\right\}$, i.e., $v_{*}$ solves

$$
\begin{cases}\partial_{t} v_{*}-\Delta v_{*}=0 & \text { in }\left\{v^{*}=0\right\}=B_{R}(0) \times[0,1]-\Omega\left(v^{*}\right), \\ v_{*}(x, 0)=u_{0}(x) & \text { on }\left\{v^{*}=0\right\} \cap\{t=0\}, \\ v_{*}=0 & \text { on } \partial\left\{v^{*}=0\right\} \cap\{t>0\} .\end{cases}
$$


Since

$$
\Omega\left(v_{*}\right)=\left\{v^{*}=0\right\} \subset\{u \geq 0\},
$$

we have $v_{*}(x, t) \leq u(x, t)$ in $\left\{v^{*}=0\right\}$. Moreover, for any given $t>0, \tilde{v}^{-}(x, s):=v^{*}(\sqrt{t} x, t s)$ satisfies the assumptions of Lemma 2.5. Thus it follows that $v^{*}(\cdot, t)$ is $t^{a}$-close to a harmonic function in $B_{\sqrt{t}}(x)$ for some $a>0$, where $x \in \Gamma_{0}$. Moreover, due to the assumption on the initial data, $\left(v_{*}\right)_{t}=\Delta v_{*} \geq-C$ at $t=0$. Also on $\Gamma\left(v_{*}\right)$,

$$
\left(v_{*}\right)_{t} /\left|D v_{*}\right|=-\left(v^{*}\right)_{t} /\left|D v^{*}\right|=-\left|D v^{*}\right| \geq-t^{(\beta-1) /(2-\alpha)} .
$$

Here the first equality follows since $\left(v_{*}\right)_{t} /\left|D v_{*}\right|$ and $-\left(v^{*}\right)_{t} /\left|D v^{*}\right|$ are the normal velocities of their respective level sets $\Gamma\left(v_{*}\right)$ and $\Gamma\left(v^{*}\right)$, but $\Gamma\left(v^{*}\right)=\Gamma\left(v_{*}\right)$ by definition. The second equality follows since $v^{*}$ solves the one phase problem (ST1), and the last inequality follows from (3-6).

Since $\Omega\left(v_{*}\right)$ is Lipschitz and $\Gamma_{t}\left(v_{*}\right)=\Gamma_{t}\left(v^{*}\right)$ is regularized in space over time (see Theorem 2.16), (3-6) also holds for $\left|D v_{*}\right|$.

Hence on $\Gamma\left(v_{*}\right)$,

$$
\left(v_{*}\right)_{t}=-\left|D v^{*}\right|\left|D v_{*}\right| \geq-t^{2(\beta-1) /(2-\alpha)}>-t^{-2 / 5},
$$

where $\alpha$ and $\beta$ are the growth rates defined in (1-2), and the last inequality follows from the assumption (I-b). Since $\left(v_{*}\right)_{t}$ solves a heat equation in $\Omega\left(v_{*}\right)$, it follows that for $x \in \Gamma_{0}$,

$$
\left(v_{*}\right)_{t} \geq-t^{-2 / 5} \quad \text { in } B_{\sqrt{t} / 2}\left(x-\sqrt{t} e_{n}\right) \times[0, t] .
$$

Then since $v_{*}\left(x-\sqrt{t} e_{n}, 0\right) \geq(\sqrt{t})^{\alpha} \geq(\sqrt{t})^{7 / 6}=t^{7 / 12}$, for $x \in \Gamma_{0}$ we have

$$
\begin{aligned}
v_{*}\left(x-\sqrt{t} e_{n}, t\right)=v_{*}\left(x-\sqrt{t} e_{n}, 0\right)+\int_{0}^{t}\left(v_{*}\right)_{t}\left(x-\sqrt{s} e_{n}, s\right) d s & \geq v_{*}\left(x-\sqrt{t} e_{n}, 0\right)-\frac{5}{3} t^{3 / 5} \\
& \geq \frac{1}{2} v_{*}\left(x-\sqrt{t} e_{n}, 0\right)+\frac{1}{2} t^{7 / 12}-\frac{5}{3} t^{3 / 5} \\
& \geq \frac{1}{2} v_{*}\left(x-\sqrt{t} e_{n}, 0\right)
\end{aligned}
$$

if $t$ is sufficiently small. It follows that

$$
u^{+}\left(x-\sqrt{t} e_{n}, 0\right)=v_{*}\left(x-\sqrt{t} e_{n}, 0\right) \leq 2 v_{*}\left(x-\sqrt{t} e_{n}, t\right) \leq 2 u^{+}\left(x-\sqrt{t} e_{n}, t\right),
$$

where the first inequality follows from (3-7).

Since $\Gamma\left(v_{*}\right)=\Gamma\left(v^{*}\right)$ is Lipschitz in a parabolic scaling, $v_{*}$ is almost harmonic. Hence $v_{*}(\cdot, t)$ is bigger than the harmonic function $\omega^{t}(x)$ in $\Omega_{t}\left(v_{*}\right) \cap B_{\sqrt{t}}(x)$ with its value

$$
\omega^{t}\left(x-\sqrt{t} e_{n}\right)=\left(C_{1}\right)^{-1} u^{+}\left(x-\sqrt{t} e_{n}, 0\right) .
$$

Note that if $0 \leq t \leq t(x, s)$, then $s<\sqrt{t}$. Hence for $0 \leq t \leq t(x, s)$,

$$
C_{1} u^{+}\left(x-s e_{n}, t\right) \geq C_{1} v_{*}\left(x-s e_{n}, t\right) \geq C_{1} \omega^{t}\left(x-s e_{n}\right) \geq C u^{+}\left(x-s e_{n}, 0\right),
$$

where the last inequality follows since the one-phase result implies a power law on the movement of $\Gamma\left(v^{*}\right)=\Gamma\left(v_{*}\right)\left(\right.$ see Lemma 2.5 of [Choi et al. 2007]), and this yields a bound on $u^{+}\left(x-s e_{n}, 0\right) / \omega^{t}\left(x-s e_{n}\right)$. 
Similar arguments apply to $u^{-}$, if we consider the function $v^{* *}$ solving (ST1) with initial data $u_{0}^{+}$, and the function $v^{\star}$ solving the heat equation in $\left\{v^{* *}=0\right\}$ with initial data $u_{0}$ and with boundary data 0 on $\Gamma\left(v^{* *}\right)$ and -1 on $\partial B_{R}(0)$.

Lemma 3.5 (distance estimate at $t=0$ ). Let $u$ be as in Theorem 1.1. Let $x \in \Gamma_{0}$ and let $e_{n}=x /|x|$ after a rotation. Let s be a sufficiently small positive constant. If

$$
\frac{\left|u^{+}\left(x-s e_{n}, 0\right)\right|}{s} \leq m \quad \text { and } \quad \frac{\left|u^{-}\left(x+s e_{n}, 0\right)\right|}{s} \leq m,
$$

then for $t \in[0, s / m]$,

$$
d(x, t)=\sup \left\{r: x+r e_{n} \text { or } x-r e_{n} \in \Gamma_{t}(u)\right\} \leq s .
$$

Proof. Let $v^{* *}$ solve (ST1) with initial data $u_{0}^{+}$, and let $v^{*}$ solve (ST1) with initial data $u_{0}^{-}$and with $v^{*}=1$ on $\partial B_{R}(0)$. Then by comparison, $-v^{*} \leq u \leq v^{* *}$ and the lemma follows from the one-phase result of Theorem 2.16.

In the next lemma, we approximate our solution by harmonic functions.

Note that, due to Lemma 3.1, We know that the rescaled function $\tilde{u}(x, t)$ as given in (1-4) satisfies the condition $\left(\mathrm{B}^{\prime}\right)$ in the space variable. On the other hand, it is not clear if the level sets of $u$ are close to Lipschitz graphs in the time variable. The approximation by harmonic functions given by Lemma 3.6, as well as Harnack-type inequalities obtained at $t=0$ and at future times, will ensure us that $\Gamma(u)$ is almost Lipschitz in the time variable as well (Corollary 4.4). This fact will serve as the first step towards the regularization procedure in Section 5.

Lemma 3.6 (spatial regularity in the whole domain). Let $u$ be as in Theorem 1.1. Then there exists a positive constant $r_{0}$ depending only on $n$ such that for $x_{0} \in \Gamma_{0}$ and $0<r<r_{0}$, there exists a function $\omega(x, t):=\omega^{+}(x, t)-\omega^{-}(x, t)$ that satisfies:

(a) $\omega(\cdot, t)$ is harmonic in its positive and negative phase in $(1+r) \Omega_{t}(u)-(1-r) \Omega_{t}(u)$, and $\Omega\left(\omega^{+}\right)$, $\Omega\left(\omega^{-}\right)$are star-shaped with respect to $B_{r_{0}}(0)$ given in $(\mathrm{I}-\mathrm{a})$.

(b) For a dimensional constant $C>0$, we have

$$
\begin{aligned}
& \omega^{+}(x, t) \leq u^{+}(x, t) \leq C \omega^{+}\left(\left(1-r^{5 / 4}\right) x, t\right) \quad \text { and } \quad \omega^{-}(x, t) \leq u^{-}(x, t) \leq C \omega^{-}\left(\left(1+r^{5 / 4}\right) x, t\right) \\
& \text { in } B_{r}\left(x_{0}\right) \times\left[r^{2}, t\left(x_{0}, r\right)\right] .
\end{aligned}
$$

Note that $t\left(x_{0}, r\right) \geq r^{7 / 6} \geq r^{2}$, and $\partial\left\{\omega^{+}>0\right\}$ need not be $\partial\left\{\omega^{-}>0\right\}$.

Proof. Step 1. We will only show the lemma for $u^{+}$. For a given $x_{0} \in \Gamma_{0}$, we may assume that $e_{n}=x_{0} /\left|x_{0}\right|$ after a rotation.

First we will construct a barrier function $v_{1}$ which will serve as a supersolution of (ST2). For this, let us first consider the viscosity solution $u^{\star}$ of (ST1) with the initial data $u_{0}^{+}$for $0 \leq t \leq t_{0}$. We may assume that for $t_{0}$ small compared to $R$ the support of $u^{\star}$ stays inside $B_{R}(0)$. Let us define

$$
\Omega_{+}^{\star}:=\left\{u^{\star}>0\right\}, \quad \Gamma^{\star}:=\partial\left\{u^{\star}=0\right\}, \quad \Omega_{-}^{\star}:=B_{R}(0) \times\left[0, t_{0}\right]-\bar{\Omega}_{+}^{\star} .
$$


Now let $v_{1}$ solve the heat equation in $\Omega_{+}^{\star}$ and in $\Omega_{-}^{\star}$, with initial data $u_{0}$ and with $v_{1}=-1$ on $\partial B_{R}(0)$. In other words, $v_{1}=v_{1}^{+}-v_{1}^{-}$, where

$$
\begin{cases}\partial_{t} v_{1}^{+}-\Delta v_{1}^{+}=0 & \text { in } \Omega_{+}^{\star}, \\ v_{1}^{+}(x, 0)=u_{0}^{+}(x) & \text { on }\{t=0\} \\ v_{1}^{+}=0 & \text { on } \Gamma^{\star}\end{cases}
$$

and

$$
\begin{cases}\partial_{t} v_{1}^{-}-\Delta v_{1}^{-}=0 & \text { in } \Omega_{-}^{\star} \\ v_{1}^{-}(x, 0)=u_{0}^{-}(x) & \text { on }\{t=0\} \\ v_{1}^{-}=0 & \text { on } \Gamma^{\star} \\ v_{1}^{-}=1 & \text { on } \partial B_{R}(0) \times[0,1] .\end{cases}
$$

Note that $v_{1}$ solves the heat equation in two regions $\Omega_{+}^{\star}$ and $\Omega_{-}^{\star}$, with free boundary $\Gamma^{\star}$. Also note that $v_{1}^{+}=u^{\star}$ and $\partial_{t} v_{1}^{+}=\left|D v_{1}^{+}\right|^{2}$ on $\Gamma^{\star}$ since the boundary $\Gamma^{\star}$ is obtained from the one phase problem with initial data $u_{0}^{+}$. Hence we can observe that $v_{1}$ is a supersolution of the two-phase problem (ST2).

Similarly one can construct a subsolution of (ST2): let us consider $\tilde{u}^{\star}$ : the viscosity solution of (ST1) in $B_{R}(0) \times\left[0, t_{0}\right]$ with the initial data $u_{0}^{-}$and fixed boundary data 1 on $\partial B_{R}(0) \times\left[0, t_{0}\right]$. Let us define

$$
\widetilde{\Omega}_{-}^{\star}:=\left\{\tilde{u}^{\star}>0\right\}, \quad \widetilde{\Gamma}^{\star}:=\partial\left\{\tilde{u}^{\star}=0\right\}, \quad \widetilde{\Omega}_{+}^{\star}:=B_{R}(0) \times\left[0, t_{0}\right]-\widetilde{\Omega}_{-}^{\star} .
$$

Now let $v_{2}$ solve the heat equation in two regions $\widetilde{\Omega}_{-}^{\star}$ and $\widetilde{\Omega}_{+}^{\star}$, with boundary data 0 on $\widetilde{\Gamma}^{\star}$ and -1 on $\partial B_{R}(0)$, and with initial data $u_{0}$. Note that $v_{2}^{-}=\tilde{u}^{\star}$. Then $v_{2}$ is a subsolution of (ST2), and by comparison,

$$
v_{2} \leq u \leq v_{1}
$$

Hence the free boundary of $u$ is trapped between the free boundaries of $v_{1}$ and $v_{2}$. Note that the free boundaries $\Gamma^{\star}$ and $\widetilde{\Gamma}^{\star}$ of $v_{1}$ and $v_{2}$ are obtained from the one-phase problem (ST1). Hence by Theorem 2.16(a), $\Gamma^{\star}$ and $\widetilde{\Gamma}^{\star}$ are Lipschitz in space in $B_{d}\left(x_{0}\right)$ for a small constant $d>0$. Also, Theorem 2.16(c) implies that for $\delta \in[d / 2, d]$ and $x_{0}+\delta e_{n} \in \Gamma_{t}^{\star}$, the normal velocity $V_{x_{0}+\delta e_{n}, t}$ of $\Gamma^{\star}$ at $\left(x_{0}+\delta e_{n}, t\right)$ satisfies

$$
V_{x_{0}+\delta e_{n}, t}=\left|D v_{1}^{+}\left(x_{0}+\delta e_{n}, t\right)\right| \sim \frac{d}{t} \sim \frac{u_{0}^{+}\left(x_{0}-d e_{n}\right)}{d} \leq d^{\beta-1} .
$$

Since $d / t \leq d^{\beta-1}$, we obtain

$$
t \geq d^{2-\beta}>d^{2}
$$

Hence the above speed bound of $\Gamma^{\star}$ implies that $\Omega_{+}^{\star}$ and $\Omega_{-}^{\star}$ are Lipschitz in space and time, in parabolic scaling. Then by Lemma 2.5, $v_{1}^{+}$and $v_{1}^{-}$are almost harmonic up to a $d$-neighborhood of their free boundaries for $t \geq d^{2}$. Similarly, we obtain that $v_{2}^{+}$and $v_{2}^{-}$are almost harmonic up to a $d$-neighborhood of their free boundaries for $t \geq d^{2}$.

Next we fix $r \leq d$. Note that if $t \leq t\left(x_{0}, r\right)$, then by Theorem 2.16(c), both of the sets $\Gamma_{t}\left(v_{1}\right)$ and $\Gamma_{t}\left(v_{2}\right)$ are within distance $r$ of $\Gamma_{0}(u)$ in $B_{r}\left(x_{0}\right)$ during this time. In particular, arguments parallel to the 
ones in the proofs of Lemmas 2.1 and 2.3 in [Choi and Kim 2010] yield that

$$
\sup \left\{u(y, s):(y, s) \in B_{d}\left(x_{0}\right) \times\left[0, d^{2}\right]\right\} \sim u\left(x-d e_{n}, 0\right) .
$$

Now using the almost harmonicity of $v_{1}^{+}$and $v_{2}^{+}$, we conclude that for $0 \leq t \leq t\left(x_{0}, r\right)$,

$$
v_{2}\left(x_{0}-2 r e_{n}, t\right) \sim u_{0}\left(x_{0}-2 r e_{n}, 0\right) \sim v_{1}\left(x_{0}-2 r e_{n}, t\right) .
$$

Step 2. Observe that by the definition of $t\left(x_{0}, r\right)$ and the assumption on the growth rates of $u_{0}$,

$$
r^{2-\beta} \leq t\left(x_{0}, r\right) \leq r^{2-\alpha} \leq r^{5 / 6}:=\tau
$$

Due to Lemma 3.1, we know that at each time, $\Omega_{t}(u)$ is $\tau^{5}$-close to a star-shaped domain $D_{t}$ up to the time $t=\tau$, i.e.,

$$
D_{t} \subset \Omega_{t}(u) \subset\left(1+\tau^{5}\right) D_{t} \subset\left(1+r^{4}\right) D_{t}
$$

for $0 \leq t \leq \tau$.

Also note that by the first inequality of (3-10) with $\beta \geq 5 / 6$,

$$
t\left(z, r^{13 / 20}\right) \geq r^{13(2-\beta) / 20}>\tau \text { for any } z \in \Gamma_{0} .
$$

Hence we can apply Lemma 3.3 for $s=r^{13 / 20}$ up to the time $\tau$. Then by Lemma 3.3 and (3-11) with $\beta \geq 5 / 6$,

$$
u(x, t) \leq r^{(13 / 20)(5 / 6)}=r^{13 / 24}
$$

for $x \in \partial\left(1-r^{13 / 20}\right) D_{0}$ and for $0 \leq t \leq \tau$. Then by the $\tau^{5}$-monotonicity of $u$,

$$
u(x, t) \leq r^{13 / 24} \text { on } B_{R}(0)-\left(1-r^{13 / 20}+r^{4}\right) D_{0}
$$

for $0 \leq t \leq \tau$. Since $\Gamma_{t}(u)$ is located between the free boundaries $\Gamma^{\star}$ and $\widetilde{\Gamma}^{\star}$ of one-phase problem, Lemma 2.12 with $\beta \geq 5 / 6$ implies that $\Gamma(u)$ stays in the $\tau^{6 / 7}$-neighborhood of $\Gamma_{0}(u)$ up to $\tau$. Also (3-11) implies that $\partial D_{t}$ stays in the $\tau^{5}$-neighborhood of $\Gamma_{t}(u)$ up to $\tau$. Hence we obtain that $\partial D_{t}$ stays in the $\tau^{5 / 6}$-neighborhood of $\partial D_{0}$ up to the time $\tau$. Since $\tau^{5 / 6}=r^{25 / 36}<r^{13 / 20},(3-12)$ implies

$$
u(x, t) \leq r^{13 / 24} \text { on } B_{R}(0)-D_{s}
$$

for any $0 \leq s, t \leq \tau$.

Step 3. Let

$$
t_{0}=0 \leq t_{1}=r^{2} \leq t_{2}=2 r^{2} \leq \cdots \leq t_{k_{0}}=k_{0} r^{2} \leq \tau
$$

and fix a number $b$ such that

$$
5 / 4 \leq b<61 / 48
$$

We will construct a supersolution of (ST2) in

$$
\left(B_{R}(0)-\left(1+r^{b}\right) D_{t_{k}}\right) \times\left[t_{k}, t_{k+1}\right] .
$$


Let $w^{k}(x)$ be the harmonic function in

$$
\Sigma:=\left(1+4 r^{b}\right) D_{t_{k}}-D_{t_{k}}
$$

with boundary data zero on $\partial\left(1+4 r^{b}\right) D_{t_{k}}$ and $C_{n} r^{13 / 24}$ on $\partial D_{t_{k}}$, where $C_{n}$ is a sufficiently large dimensional constant. Extend $w^{k}(x)$ by 0 to $\mathbb{R}^{n}-\Sigma$. Next define

$$
\Phi^{k}(x, t):=\inf \left\{w^{k}(y):|x-y| \leq r^{b}-\left(t-t_{k}\right) \frac{1}{2} r^{b-2}\right\}
$$

in $\left(B_{R}(0)-\left(1+r^{b}\right) D_{t_{k}}\right) \times\left[t_{k}, t_{k+1}\right]$. We claim that the function $\Phi^{k}$ is a supersolution of (ST2) in $\left(B_{R}(0)-\left(1+r^{b}\right) D_{t_{k}}\right) \times\left[t_{k}, t_{k+1}\right]$, since our constant $b$ satisfies

$$
r^{b-2}>r^{13 / 24-b} .
$$

For simplicity, write $\Phi=\Phi^{k}$. To check that $\Phi$ is a supersolution, first note that $\Phi(\cdot, t)$ is superharmonic in its positive set and $\Phi_{t} \geq 0$. Hence we only need to show that

$$
\frac{\Phi_{t}}{|D \Phi|} \geq|D \Phi| \quad \text { on } \Gamma(\Phi) \text {. }
$$

Due to the definition of $\Phi, \Gamma_{t}(\Phi)$ has an interior ball of radius at least $r^{b} / 2$ for $t_{k} \leq t \leq t_{k+1}$. This and the superharmonicity of $\Phi$ in the positive set yield that

$$
|D \Phi| \leq \frac{C r^{13 / 24}}{r^{b}} \text { on } \Gamma(\Phi)
$$

for a dimensional constant $C>0$. Moreover $\Gamma(\Phi)$ evolves with normal velocity $\frac{1}{2} r^{b-2}$. Since (3-14) holds for our choice of $b$ (i.e., for $5 / 4 \leq b<61 / 48$ ), we conclude that (3-15) holds for $r$ smaller than a dimensional constant $r(n)$. Now we compare $u$ with $\Phi$ on

$$
\left(B_{R}(0)-\left(1+r^{b}\right) D_{t_{k}}\right) \times\left[t_{k}, t_{k+1}\right] .
$$

Note that by (3-13),

$$
u^{+} \leq \Phi \quad \text { on } \partial\left(1+r^{b}\right) D_{t_{k}}
$$

if $C_{n}$ is chosen sufficiently large. Also at $t=t_{k},(3-11)$ implies

$$
u\left(\cdot, t_{k}\right) \leq 0 \leq \Phi\left(\cdot, t_{k}\right) \quad \text { on } B_{R}(0)-\left(1+r^{b}\right) D_{t_{k}} .
$$

Hence we get $u \leq \Phi$ in $\left(\mathbb{R}^{n}-\left(1+r^{b}\right) D_{t_{k}}\right) \times\left[t_{k}, t_{k+1}\right]$. This implies

$$
\Omega(u) \subset \Omega(\Phi) \cup\left(\left(1+r^{b}\right) D_{t_{k}} \times\left[t_{k}, t_{k+1}\right]\right):=\widetilde{\Omega}(\Phi)
$$

for $t_{k} \leq t \leq t_{k+1}$.

Step 4. Next we let $v(x, t)$ solve the heat equation in

$$
\widetilde{\Omega}(\Phi)-\left((1-3 r) \Omega_{0}(u) \times\left[t_{k}, t_{k+1}\right]\right)
$$


with initial data $v\left(\cdot, t_{k}\right)=u\left(\cdot, t_{k}\right)$ and boundary data zero on $\Gamma(\Phi)$ and $v=u$ on $(1-3 r) \Gamma_{0}(u)$. Observe that, due to (3-16), we have

$$
u^{+} \leq v \quad \text { for } t_{k} \leq t \leq t_{k+1}
$$

Since $\widetilde{\Omega}(\Phi)$ is star-shaped and expands with its normal velocity $<r^{b-2}$, which is less than $r^{-1}$, Lemma 2.5 applies to $\tilde{v}(x, t):=v\left(r x, r^{2} t\right)$. In particular there exists a constant $C>0$ such that

$$
(1 / C) v(x, t) \leq h_{1}(x, t) \leq C v(x, t)
$$

for $\left(t_{k}+t_{k+1}\right) / 2 \leq t \leq t_{k+1}$, where $h_{1}(\cdot, t)$ is the harmonic function in $\Omega_{t}(v)-(1-2 r) \Omega_{0}(u)$ with boundary data zero on $\Gamma_{t}(v)$ and $v$ on $(1-2 r) \Gamma_{0}(u)$.

Hence we conclude that

$$
u^{+} \leq v \leq C h_{1}
$$

in $\left(B_{R}(0)-(1-2 r) \Omega_{0}(u)\right) \times\left[\left(t_{k}+t_{k+1}\right) / 2, t_{k+1}\right]$.

Step 5. Similar arguments, now pushing the boundary purely by the minus phase given by the harmonic function, yield that

$$
\Pi_{t}:=\left\{x \in D_{t_{k}}: \operatorname{dist}\left(x, \partial D_{t_{k}}\right) \geq 3 r^{b}+\frac{1}{2} r^{b-2}\left(t-t_{k}\right)\right\} \subset \Omega_{t}(u)
$$

for $t_{k} \leq t \leq t_{k+1}$. Let $w(x, t)$ solve the heat equation in

$$
\left.\Pi-\left((1-3 r) \Omega_{0}(u) \times\left[t_{k}, t_{k+1}\right]\right)\right)
$$

with initial data $u\left(\cdot, t_{k}\right)$ and boundary data zero on $\partial \Pi$, and $u$ on $(1-3 r) \Gamma_{0}(u)$. Then $u \geq w(x, t)$. Since $\Pi$ is star-shaped and it shrinks with its normal velocity $<r^{b-2}$, which is less than $r^{-1}$, Lemma 2.5 applies to $\tilde{w}(x, t):=w\left(r x, r^{2} t\right)$. In particular there exists $C>0$ such that

$$
u^{+} \geq w \geq(1 / C) h_{2}
$$

for $\left(t_{k}+t_{k+1}\right) / 2 \leq t \leq t_{k+1}$, where $h_{2}(\cdot, t)$ is the harmonic function in $\Pi_{t}-(1-2 r) \Omega_{0}(u)$ with boundary data coinciding with that of $w$.

Step 6. Lastly we will show that $h_{1}$ and $h_{2}$ are not too far apart, i.e.,

$$
h_{1}(x, t) \leq C h_{2}\left(x-8 r^{b} e_{n}, t\right),
$$

with a dimensional constant $C>0$. Since $u$ is between $(1 / C) h_{2}$ and $C h_{1}$, this will conclude our proof for $\left(t_{k}+t_{k+1}\right) / 2 \leq t \leq t_{k+1}$. Then by changing the time intervals $\left[t_{k}, t_{k+1}\right]$ to $\left[t_{k}+r^{2} / 2, t_{k+1}+r^{2} / 2\right]$, we obtain the lemma for any $t \in\left[r^{2}, t\left(x_{0}, r\right)\right]$.

To prove (3-18), observe that by the construction of $v$ and $w$,

$$
\Omega_{t}(w) \subset \Omega_{t}(v) \subset\left(1+8 r^{b}\right) \Omega_{t}(w) .
$$

Since $t_{k+1}-t_{k}=r^{2}$, Lemma 2.12 implies

$$
\sup \left\{d\left(x, \Gamma_{t}(u)\right): x \in \Gamma_{t_{k}}(u)\right\} \leq r^{12 / 7}
$$


for $t \in\left[t_{k}, t_{k+1}\right]$. Then by (3-11),

$$
\sup \left\{d\left(x, \Gamma_{t}(u)\right): x \in \partial D_{t_{k}}\right\} \leq r^{12 / 7}+r^{4} \ll r^{b}
$$

for $t \in\left[t_{k}, t_{k+1}\right]$. Then we obtain

$$
v_{2}(x, t) \leq v(x, t) \leq v_{1}\left(\left(1-4 r^{b}\right) x,\left(1-4 r^{b}\right)^{2}\left(t-t_{k}\right)+t_{k}\right)
$$

for $t_{k} \leq t \leq t_{k+1}$, where the first inequality follows from (3-8) and (3-17), and the second inequality follows from the comparison principle along with (3-8), $v\left(\cdot, t_{k}\right)=u\left(\cdot, t_{k}\right)$ and (3-19). Similarly,

$$
v_{2}\left(\left(1+4 r^{b}\right) x,\left(1+4 r^{b}\right)^{2}\left(t-t_{k}\right)+t_{k}\right) \leq w(x, t) \leq v_{1}(x, t) .
$$

Combing (3-20) and (3-21), we get

$$
\begin{aligned}
v_{2}\left(\left(1+4 r^{b}\right) x,\left(1+4 r^{b}\right)^{2}\left(t-t_{k}\right)+t_{k}\right) & \leq w(x, t), v(x, t) \\
& \leq v_{1}\left(\left(1-4 r^{b}\right) x,\left(1-4 r^{b}\right)^{2}\left(t-t_{k}\right)+t_{k}\right) .
\end{aligned}
$$

This and (3-9) yield

$$
v\left(x_{0}-2 r e_{n}, t\right) \sim w\left(x_{0}-2 r e_{n}, t\right) \sim u\left(x_{0}-2 r e_{n}, 0\right) .
$$

It follows that

$$
w(x, t) \leq v(x, t) \leq C w\left(x-8 r^{b} e_{n}, t\right) \quad \text { on }(1-2 r) \Gamma_{0} \times\left[t_{k}, t_{k+1}\right] .
$$

Hence due to Dahlberg's lemma, we conclude that

$$
h_{1}(x, t) \leq C_{1} v(x, t) \leq C_{2} w\left(x-8 r^{b} e_{n}, t\right) \leq C_{3} h_{2}\left(x-8 r^{b} e_{n}, t\right)
$$

in $B_{r}\left(x_{0}\right) \times\left[\left(t_{k}+t_{k+1}\right) / 2, t_{k+1}\right]$. Since the inequality holds for any $5 / 4 \leq b<61 / 48$, we obtain the lemma.

Next we show that in the "unbalanced" region, where one phase has much larger flux than the other, the regularization process occurs similarly to the one in the one-phase problem. This observation will be useful for the analysis in Section 4.

Proposition 3.7 (regularization in unbalanced region I). Let $u$ be as given in Theorem 1.1. For a fixed $x_{0} \in \Gamma_{0}(u)$, we may let $e_{n}=x_{0} /\left|x_{0}\right|$ after a rotation. Suppose that either

$$
u^{+}\left(x_{0}-r e_{n}, 0\right) \geq M u^{-}\left(x_{0}+r e_{n}, 0\right) \quad \text { or } \quad u^{-}\left(x_{0}+r e_{n}, 0\right) \geq M u^{+}\left(x_{0}-r e_{n}, 0\right)
$$

for $M>M_{n}$, where $M_{n}$ is a sufficiently large dimensional constant. Then, for $r \leq 1 / M_{n}$, there exists a dimensional constant $C>0$ such that

$$
\left|D u^{+}(x, t)\right| \leq C \frac{u^{+}\left(x_{0}-r e_{n}, 0\right)}{r} \quad \text { and } \quad\left|D u^{-}(x, t)\right| \leq C \frac{u^{-}\left(x_{0}+r e_{n}, 0\right)}{r}
$$

in $B_{r}\left(x_{0}\right) \times\left[t\left(x_{0}, r\right) / 2, t\left(x_{0}, r\right)\right]$. 
Remark 3.8. 1. In the next section, we will extend Proposition 3.7 to later times, i.e., to $x_{0} \in \Gamma_{t_{0}}$ (see Lemma 4.7).

2. The situation given in Proposition 3.7 is essentially a perturbation of the one-phase case in [Choi and Kim 2010]. The main step in the proof is the verification of this observation; by barrier arguments we will show that our solution is very close to a rescaled version of the one-phase solution, for which the regularity of solutions is well-understood (see Theorem 2.16).

Proof of Proposition 3.7. Without loss of generality, we may assume that

$$
u^{+}\left(x_{0}-r e_{n}, 0\right) \geq M u^{-}\left(x_{0}+r e_{n}, 0\right) .
$$

Step 1. First we will show that after a small amount of time $u$ becomes almost harmonic near the free

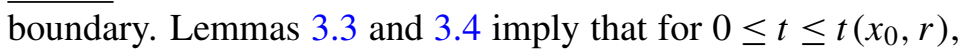

$$
u^{+}\left(x_{0}-r e_{n}, t\right) \sim u^{+}\left(x_{0}-r e_{n}, 0\right), \quad u^{-}\left(x_{0}+r e_{n}, t\right) \sim u^{-}\left(x_{0}+r e_{n}, 0\right) .
$$

Also note that, by the assumption on the initial data $u_{0}$, Lemma 3.6 holds at $t=0$. In other words, there exists a function $\omega(x, 0)=\omega_{0}(x)$ such that:

(a) $\omega_{0}$ is harmonic in its positive and negative phases in $(1+r) \Omega_{0}(u)-(1-r) \Omega_{0}(u)$.

(b) $\Omega\left(\omega_{0}^{+}\right)$and $\Omega\left(\omega_{0}^{-}\right)$are star-shaped.

(c) In $B_{r}\left(x_{0}\right)$, we have

$$
\begin{aligned}
& \omega_{0}^{+}(x) \leq u_{0}^{+}(x) \leq C \omega_{0}^{+}\left(\left(1-r^{5 / 4}\right) x\right), \\
& \omega_{0}^{-}(x) \leq u_{0}^{-}(x) \leq C \omega_{0}^{-}\left(\left(1+r^{5 / 4}\right) x\right) .
\end{aligned}
$$

Next we will improve (3-23) and (3-24) for later times to obtain the inequalities with $C=\left(1+r^{a}\right)$ for $t \geq r^{3 / 2}$. By the distance estimate in Lemma 2.12, the free boundary of $u$ moves less that $r^{9 / 7}<r^{5 / 4}$ during the time $t=r^{3 / 2}$. Then we let $v_{1}$ solve

$$
\begin{cases}\partial_{t} v_{1}=\Delta v_{1} & \text { in }\left(1+2 r^{5 / 4}\right) \Omega_{0}\left(\omega^{+}\right) \times\left[0, r^{3 / 2}\right], \\ \partial_{t} v_{1}=\Delta v_{1} & \text { in }\left(B_{R}(0)-\left(1+2 r^{5 / 4}\right) \bar{\Omega}_{0}\left(\omega^{+}\right)\right) \times\left[0, r^{3 / 2}\right], \\ v_{1}(\cdot, 0)=u_{0}^{+} & \text {on }\left(1+2 r^{5 / 4}\right) \Omega_{0}\left(\omega^{+}\right), \\ v_{1}(\cdot, 0)=-u_{0}^{-} & \text {on } B_{R}(0)-\left(1+2 r^{5 / 4}\right) \bar{\Omega}_{0}\left(\omega^{+}\right), \\ v_{1}=0 & \text { on }\left(1+2 r^{5 / 4}\right) \Gamma_{0}\left(\omega^{+}\right) \times\left[0, r^{3 / 2}\right], \\ v_{1}=-1 & \text { on } \partial B_{R}(0) \times\left[0, r^{3 / 2}\right] .\end{cases}
$$

Similarly, we let $v_{2}$ solve the heat equation in two cylindrical regions,

$$
\left(1-2 r^{5 / 4}\right) \Omega_{0}\left(\omega^{+}\right) \times\left[0, r^{3 / 2}\right], \quad \text { and } \quad\left(B_{R}(0)-\left(1-2 r^{5 / 4}\right) \bar{\Omega}_{0}\left(\omega^{+}\right)\right) \times\left[0, r^{3 / 2}\right]
$$

with initial data $u_{0}^{+}$and $-u_{0}^{-}$, and with lateral boundary data zero on $\left(1-2 r^{5 / 4}\right) \Gamma_{0}\left(\omega^{+}\right) \times\left[0, r^{3 / 2}\right]$ and -1 on $\partial B_{R}(0) \times\left[0, r^{3 / 2}\right]$. Then, by comparison,

$$
v_{2}<u<v_{1} \text {. }
$$


Also by Lemma 2.5 with $\beta \geq 5 / 6$,

$$
\left|v_{1}-v_{2}\right| \leq r^{5 / 4 \times 5 / 6}=r^{25 / 24} .
$$

Note that on $\left(1-r^{6 / 7}\right) \Gamma_{0}\left(\omega^{+}\right)$,

$$
\left|v_{1}\right| \geq r^{(6 / 7) \alpha} \geq r^{6 / 7 \times 7 / 6}=r
$$

and thus for $a_{1}=1 / 24$,

$$
\left|v_{1}-v_{2}\right| \leq r^{a_{1}}\left|v_{1}\right| \quad \text { on }\left(1-r^{6 / 7}\right) \Gamma_{0}\left(\omega^{+}\right)
$$

Similarly,

$$
\left|v_{1}-v_{2}\right| \leq r^{a_{1}}\left|v_{2}\right| \quad \text { on }\left(1+r^{6 / 7}\right) \Gamma_{0}\left(\omega^{+}\right)
$$

Now, $v_{1}$ and $v_{2}$ are almost harmonic in the $r^{3 / 4}$-neighborhood of their boundaries for $\frac{1}{2} r^{3 / 2} \leq t \leq r^{3 / 2}$ by Lemma 2.5. Then the almost harmonicity of $v_{1}$ and $v_{2}$ with (3-25)-(3-27) imply the following: For $\frac{1}{2} r^{3 / 2} \leq t \leq r^{3 / 2}$, there exist positive harmonic functions $\widetilde{\omega}^{+}(\cdot, t)$ and $\widetilde{\omega}^{-}(\cdot, t)$ defined respectively in

$$
\left.\Omega_{t}\left(v_{2}^{+}\right) \cap\left(B_{R}(0)-\left(1-r^{1-b}\right) \Omega_{0}\left(\omega^{+}\right)\right) \quad \text { and } \quad \Omega_{t}\left(v_{1}^{-}\right) \cap\left(1+r^{1-b}\right) \Omega_{0}\left(\omega^{+}\right)\right) \text {, }
$$

where $b=1 / 7$, such that for some $a>0$,

$$
\widetilde{\omega}^{+}(x, t) \leq u^{+}(x, t) \leq\left(1+r^{a}\right) \widetilde{\omega}^{+}\left(\left(1-4 r^{5 / 4}\right) x, t\right)
$$

and

$$
\widetilde{\omega}^{-}(x, t) \leq u^{-}(x, t) \leq\left(1+r^{a}\right) \widetilde{\omega}^{-}\left(\left(1+4 r^{5 / 4}\right) x, t\right)
$$

Now on the time interval $\left[0, r^{3 / 2}\right]+\frac{1}{2} k r^{3 / 2}, 1 \leq k \leq m$, we construct $v_{1}$ and $v_{2}$ so that they solve the heat equation in the cylindrical domains with

$$
\begin{aligned}
& \Gamma\left(v_{1}\right)=\left(1+2 r^{5 / 4}\right) \Gamma_{\frac{1}{2} k r^{3 / 2}}\left(\omega^{+}\right) \times\left[\frac{1}{2} k r^{3 / 2},\left(1+\frac{1}{2} k\right) r^{3 / 2}\right], \\
& \Gamma\left(v_{2}\right)=\left(1-2 r^{5 / 4}\right) \Gamma_{\frac{1}{2} k r^{3 / 2}}\left(\omega^{+}\right) \times\left[\frac{1}{2} k r^{3 / 2},\left(1+\frac{1}{2} k\right) r^{3 / 2}\right] .
\end{aligned}
$$

By a similar argument to the one above, we then obtain harmonic functions $\widetilde{\omega}^{ \pm}(\cdot, t)$ satisfying $(3-28)$ and (3-29) for

$$
\frac{1}{2}(1+k) r^{3 / 2} \leq t \leq\left(1+\frac{1}{2} k\right) r^{3 / 2}
$$

Hence we conclude (3-28) and (3-29) for $r^{3 / 2} \leq t \leq t\left(x_{0}, r\right)$.

Step 2. Next we rescale $u(x, t)$ as

$$
\tilde{u}(x, t):=\alpha^{-1} u\left(r x+x_{0}, r^{2} \alpha^{-1} t\right) \quad \text { in } 2 Q_{x_{0}},
$$


where $\alpha:=u^{+}\left(x_{0}-r e_{n}, 0\right) \ll r^{1 / 2}$. Then $\tilde{u}(x, t)$ solves

$$
\begin{cases}\left(\alpha \partial_{t}-\Delta\right) \tilde{u}=0 & \text { in } \Omega(\tilde{u}), \\ V=\left|D \tilde{u}^{+}\right|-\left|D \tilde{u}^{-}\right| & \text {on } \Gamma(\tilde{u}), \\ \tilde{u}\left(-e_{n}, 0\right)=1, & \\ \tilde{u}\left(e_{n}, 0\right)=-1 / N, & \text { where } N \geq M .\end{cases}
$$

Furthermore, (3-22) implies that for $0 \leq t \leq 1$,

$$
\tilde{u}^{+}\left(-e_{n}, t\right) \sim 1, \quad \tilde{u}^{-}\left(e_{n}, t\right) \sim 1 / N
$$

Let $\tilde{w}$ be the corresponding rescaled version of $\tilde{\omega}$ given in (3-28) and (3-29), then in $B_{r^{-b}}(0) \cap \Omega_{0}(\tilde{u})$ we have

$$
\left(1-r^{a}\right) \tilde{w}^{+}\left(\left(1+4 r^{5 / 4}\right) x, \alpha r^{-1 / 2}\right) \leq \tilde{u}^{+}\left(x, \alpha r^{-1 / 2}\right) \leq \tilde{w}^{+}\left(x, \alpha r^{-1 / 2}\right)
$$

and

$$
\left(1-r^{a}\right) \tilde{w}^{-}\left(x, \alpha r^{-1 / 2}\right) \leq \tilde{u}^{-}\left(x, \alpha r^{-1 / 2}\right) \leq \tilde{w}^{-}\left(\left(1+4 r^{5 / 4}\right) x, \alpha r^{-1 / 2}\right) .
$$

Here note that

$$
\alpha r^{-1 / 2}=\sqrt{r} \cdot \frac{u^{+}\left(x_{0}-r e_{n}, t_{0}\right)}{r} \leq r^{1 / 3} .
$$

Lastly, for given $x_{0} \in \Gamma(\tilde{u}) \cap B_{1}(0)$, an argument similar to the one in (3-7) implies that

$$
\tilde{u}(x, t) \leq\left(1+r^{b}\right) \tilde{u}(x, 0) \quad \text { in } \partial B_{(1 / 2) r^{-b}}\left(r^{-b} e_{n}\right) \times[0,1] .
$$

Step 3. We claim that we can construct a supersolution $U_{1}$ and a subsolution $U_{2}$ of (ST2) such that

$$
U_{2}(x, t) \leq \tilde{u}(x, t) \leq U_{1}(x, t) \leq U_{2}\left(x-\sqrt{\epsilon} e_{n}, t\right) \quad \text { in } B_{1}(0) \times\left[\alpha r^{-1 / 2}, 1\right],
$$

and so that $U_{2}$ is a smooth solution with uniformly Lipschitz boundary in space and time. Then for sufficiently small $r>0$ the lemma will follow from analysis parallel to that of [Athanasopoulos et al. 1998].

To illustrate the main ideas, let us first assume that:

(a) (3-30) and (3-31) hold in the entire ring domain $\mathscr{R} \times[0,1]$, where

$$
\mathscr{R}:=\left\{x: d\left(x, \Gamma_{0}(\tilde{u})\right) \leq r^{-b}\right\}
$$

(b) $\tilde{u}(x, t) \leq\left(1+r^{b}\right) \tilde{u}(x, 0)$ on $\partial \mathscr{R} \times[0,1]$.

Let

$$
\Sigma:=\left\{x: d\left(x, \mathbb{R}^{n}-\Omega_{0}\right) \leq r^{-b}\right\} \times\left[\alpha r^{-1 / 2}, 1\right],
$$


and let $U_{1}^{+}$be the solution of the one-phase Hele-Shaw problem in $\Sigma$,

$$
\begin{cases}\Delta U_{1}^{+}=0 & \text { in }\left\{U_{1}^{+}>0\right\} \cap \Sigma, \\ \partial_{t} U_{1}^{+}=\left|D U_{1}^{+}\right|^{2} & \text { on } \partial\left\{U_{1}^{+}>0\right\} \cap \Sigma, \\ U_{1}^{+}\left(x, \alpha r^{-1 / 2}\right)=\tilde{w}^{+}\left(x, \alpha r^{-1 / 2}\right), & \\ U_{1}^{+}(x, t)=\left(1+r^{b}\right) \tilde{u}(x, 0) & \text { for } x \in \partial \Sigma .\end{cases}
$$

Let

$$
U_{1}=U_{1}^{+}-U_{1}^{-} \quad \text { in } \mathscr{R} \times\left[\alpha r^{-1 / 2}, 1\right],
$$

where $U_{1}^{-}(\cdot, t)$ is the harmonic function in $\mathscr{R}-\Omega\left(U_{1}^{+}\right)$with boundary data

$$
U_{1}^{-}=0 \quad \text { on } \Gamma\left(U_{1}^{+}\right), \quad U_{1}^{-}=C / N \quad \text { on } \partial \mathscr{R}-\Omega\left(U_{1}^{+}\right) .
$$

Then $U_{1}$ is a supersolution of (ST2) in $\Sigma$, and thus by Lemma 2.11 and the assumptions (a)-(b) we have $\tilde{u} \leq U_{1}$ in $\Sigma$.

Step 4. The construction of the subsolution $U_{2}$ is a bit less straightforward. We use

$$
U_{2}^{+}(x, t):=(1-\epsilon) \sup _{|y-x| \leq \sqrt{\epsilon}(1-c(t))} U_{1}^{+}((1+\sqrt{\epsilon}) y, t)
$$

where $\epsilon=1 / N$ and $c(t):=t^{4 / 5}$. Then we define

$$
U_{2}=U_{2}^{+}-U_{2}^{-} \quad \text { in } R \times\left[\alpha r^{-1 / 2}, 1\right]
$$

where $\mathscr{R}$ is the ring domain as given above and $U_{2}^{-}(\cdot, t)$ is the harmonic function in $R-\Omega\left(U_{2}^{+}\right)$with fixed boundary data zero on $\Gamma\left(U_{2}^{+}\right)$and $C / N$ on $\partial \mathscr{R}-\Omega\left(U_{2}^{+}\right)$. Then $U_{2}$ satisfies the free boundary condition

$$
V_{U_{2}} \leq(1+\epsilon)\left|D U_{2}^{+}\right|-\sqrt{\epsilon} c^{\prime}(t)
$$

Therefore, $U_{2}$ is a subsolution of (ST2) if we can show that

$$
\sqrt{\epsilon} c^{\prime}(t) \geq \epsilon\left|D U_{2}^{+}\right|+\left|D U_{2}^{-}\right| \quad \text { on } \Gamma\left(U_{2}\right)
$$

and $\int_{0}^{1} c^{\prime}(s) d s \leq 1$

The analysis performed in [Choi and Kim 2010], as in the proof of (c) of Theorem 2.16, yields that at a fixed time $t, \Gamma\left(U_{1}\right)$ regularizes in the scale of $d:=d(t)$ that solves

$$
t=\frac{d^{2}}{U_{1}\left(-d e_{n}, 0\right)} \text {. }
$$

Therefore,

$$
\left|D U_{2}^{+}\right| \sim \frac{U_{2}^{+}\left(-d e_{n}, 0\right)}{d} \text { and }\left|D U_{2}^{-}\right| \sim \frac{U_{2}^{-}\left(d e_{n}, 0\right)}{d}
$$

on

$$
\Gamma\left(U_{2}\right) \times[t / 2, t]
$$


Observe that since $\beta \geq 5 / 6$,

$$
U_{2}^{+}\left(-d e_{n}, 0\right) \leq d^{5 / 6} \quad \text { and } \quad U_{2}^{-}\left(d e_{n}, 0\right) \leq \epsilon d^{5 / 6},
$$

then we have

$$
\epsilon \frac{U_{2}^{+}\left(-d e_{n}, 0\right)}{d}+\frac{U_{2}^{-}\left(d e_{n}, 0\right)}{d} \leq \epsilon d^{-1 / 6} \leq \sqrt{\epsilon} t^{-1 / 5} .
$$

where the last inequality follows from

$$
t=d^{2} / U_{1}\left(-d e_{n}, 0\right) \leq d^{2} / d^{\alpha} \leq d^{5 / 6} .
$$

Hence $c(t)=t^{4 / 5}$ satisfies (3-33), and we conclude that $U_{2}$ is a subsolution of (ST2) in $\Sigma$.

Now we can use the fact that

$$
U_{2} \leq \tilde{u} \leq U_{1} \quad \text { in } B_{c}(0) \times\left[\alpha r^{-1 / 2}, c\right]
$$

to conclude that $\tilde{u}$ is $\sqrt{\epsilon}$ - close to a Lipschitz (and smooth) solution $U_{1}$ in $B_{1}(0) \times[1 / 2,1]$, confirming $\left(\mathrm{B}^{\prime}\right)$. Moreover (A) holds due to Lemma 3.3 and Lemma 3.4. Once we can confirm this, we can conclude our proof by using the results of [Athanasopoulos et al. 1998] with the choice of a sufficiently small $\epsilon$.

Step 5. Now we proceed to the general proof without the simplified assumptions (a) and (b) in Step 3, which are replaced by the local inequalities (3-30)-(3-32). For this we need to perturb the initial data outside of $B_{1}(0)$ (see Section 4, pages 2781-2783 of [Choi et al. 2009]), to obtain functions $W_{1}(x)$ and $W_{2}(x)$ that satisfy:

(a) $\left\{W_{k}>0\right\}$ with $k=1,2$ is star-shaped and coincides with $\Omega_{\alpha r^{-1 / 2}}(\tilde{w})$ in $B_{r^{-b}}(0)$.

(b) $\left\{W_{2}>0\right\} \subset \Omega_{\alpha r^{-1 / 2}}(\tilde{w}) \subset\left\{W_{1}>0\right\}$.

(c) $d\left(x,\left\{W_{k}>0\right\}\right) \geq r^{-b}$ with $k=1,2$ for $x \in \Gamma_{\alpha r^{-1 / 2}}(\tilde{w}) \cap\left(\mathbb{R}^{n}-B_{2 r^{-b}}(0)\right)$.

(d) $W_{k}$ is harmonic in $\left\{W_{k}>0\right\}-K$ with boundary data zero on $\Gamma\left(W_{k}\right)$ and $\left(1+r^{b}\right) \tilde{w}\left(x, \alpha r^{-1 / 2}\right)$ on $\partial K$, where

$$
K=\left\{x: d\left(x, \Gamma\left(W_{k}\right)\right) \geq r^{-b}\right\} .
$$

Let $U_{k}$ be the solution of Hele-Shaw problem in

$$
\mathbb{R}^{n}-\frac{1}{2}\left\{W_{k}>0\right\} \times\left[\alpha r^{-1 / 2}, 1\right]
$$

with initial data $W_{1}$ and with lateral boundary data $\left(1+r^{b}\right) \tilde{w}\left(x, \alpha r^{-1 / 2}\right)$. Due to Proposition 4.1 of [Choi et al. 2009], for sufficiently small $r>0$, the level sets of $U_{1}$ are then $\epsilon c$-close to those of $U_{2}$ in $B_{1}(0) \times[0,1]$. Hence we can use $U_{2}$ instead of $U_{1}$ in Step 4 and proceed as in Step 4 to conclude.

\section{Decomposition based on local phase dynamics}

Throughout the rest of the paper, let $u$ be as in Theorem 1.1, and fix $x_{0} \in \Gamma_{0}$ and a sufficiently small constant $r>0$. We will prove the regularization of the solution $u$ in $B_{r}\left(x_{0}\right) \times\left[t\left(x_{0}, r\right) / 2, t\left(x_{0}, r\right)\right]$. After a rotation if necessary, we may assume that $x_{0} /\left|x_{0}\right|=e_{n}$. 
Let us fix a constant $M \geq M_{n}$, where $M_{n}$ is a sufficiently large dimensional constant. If the ratio between $u^{+}\left(x_{0}-r e_{n}, 0\right)$ and $u^{-}\left(x_{0}+r e_{n}, 0\right)$ is bigger than $M$, then we can directly apply Proposition 3.7 to prove the main theorem. Therefore we assume that

$$
M^{-1} u^{-}\left(x_{0}+r e_{n}, 0\right) \leq u^{+}\left(x_{0}-r e_{n}, 0\right) \leq M u^{-}\left(x_{0}+r e_{n}, 0\right) .
$$

Let

$$
C_{0}:=\max \left\{\frac{u^{+}\left(x_{0}-r e_{n}, 0\right)}{r}, \frac{u^{-}\left(x_{0}+r e_{n}, 0\right)}{r}\right\} .
$$

Then since $u_{0}^{+}$and $u_{0}^{-}$are comparable with harmonic functions, $C_{0}$ is less than a constant depending on $n$ and $M$ (See Corollary 2.15). Also note that

$$
C_{0} \geq r^{\alpha-1} \geq r^{1 / 6}
$$

Let us now sort out the initial free boundary points where the flux from one phase dominates the flux from the other phase. Let us define

$$
\begin{aligned}
& A^{+}=\left\{x \in \Gamma_{0} \cap B_{2 r}\left(x_{0}\right): \frac{u^{+}\left(x-s e_{n}, 0\right)}{s} \geq M C_{0} \text { for some } s \text { with } r^{5 / 4} \leq s \leq r\right\}, \\
& A^{-}=\left\{x \in \Gamma_{0} \cap B_{2 r}\left(x_{0}\right): \frac{u^{-}\left(x+s e_{n}, 0\right)}{s} \geq M C_{0} \text { for some } s \text { with } r^{5 / 4} \leq s \leq r\right\} .
\end{aligned}
$$

We then write

$$
A=A^{+} \cup A^{-} .
$$

Throughout the paper we will let $e_{n}=x /|x|$ for any boundary point $x$, after a necessary rotation.

Lemma 4.1. Let $u$ be as given in Theorem 1.1, and let $M$ and $C$ as given above.

(a) If $\frac{u^{+}\left(x-s e_{n}, 0\right)}{s} \geq M C_{0}$ for some $s \leq r$, then $\frac{u^{+}\left(x-s e_{n}, 0\right)}{s} \leq C_{0}$.

(b) If $\frac{u^{-}\left(x+s e_{n}, 0\right)}{s} \geq M C_{0}$ for some $s \leq r$, then $\frac{u^{-}\left(x+s e_{n}, 0\right)}{s} \leq C_{0}$.

Proof. Since $u_{0}^{ \pm}$are comparable with harmonic functions $h^{ \pm}$, we can argue similarly as in Corollary 2.15. Observe that

$$
\frac{u_{0}^{+}\left(x-s e_{n}\right)}{s} \cdot \frac{u_{0}^{-}\left(x+s e_{n}\right)}{s} \sim \frac{h^{+}\left(x-s e_{n}\right)}{s} \cdot \frac{h^{-}\left(x+s e_{n}\right)}{s} \lesssim \sqrt{\phi(r)} \lesssim C_{0}^{2} .
$$

Now for $x \in A^{+}$, there exists a largest constant $r_{x}<r$ such that

$$
\frac{u^{+}\left(x-r_{x} e_{n}, 0\right)}{r_{x}}=M C_{0}
$$

We then define

$$
Q_{x}=B_{r_{x}}(x) \times\left[0, \frac{r_{x}}{M C_{0}}\right] .
$$




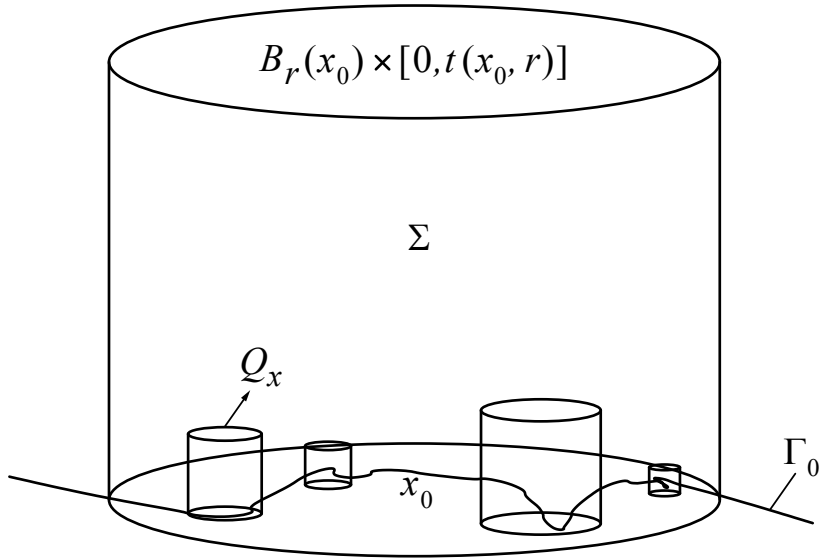

Figure 3. Decomposition of the domain.

Also for $x \in A^{-}$, we can similarly define $r_{x}$ and $Q_{x}$. Now we define

$$
\Sigma:=B_{r}\left(x_{0}\right) \times\left[0, t\left(x_{0}, r\right)\right]-\bigcup_{x \in A} Q_{x} ;
$$

see Figure 3. $\Sigma$ is then the region where the fluxes from both sides are initially balanced. Our aim in this section is to prove that the balance is kept over time, so that the interface remains close to a Lipschitz graph over time.

The following statement is a direct consequence of the definition of $\Sigma$.

Lemma 4.2. If $x \in \Gamma_{0} \cap \Sigma_{0}$, then for all $r^{5 / 4} \leq s \leq r$,

$$
\frac{u^{+}\left(x-s e_{n}, 0\right)}{s}, \frac{u^{-}\left(x+s e_{n}, 0\right)}{s} \leq M C_{0} .
$$

The next proposition, the main result in this section, states that the solution is "well-behaved" in $\Sigma$.

Proposition 4.3. There exists a dimensional constant $K>0$ such that for all $(x, t) \in \Gamma \cap \Sigma$,

$$
\frac{u^{+}\left(x-s e_{n}, t\right)}{s}, \frac{u^{-}\left(x+s e_{n}, t\right)}{s}<K M C_{0} \quad \text { for } r^{5 / 4} \leq s \leq r .
$$

Before proving Proposition 4.3, we show an immediate consequence of it; we are ready to show that $\Gamma(u)$ is close to a Lipschitz graph in time as well as in space.

Corollary 4.4. For $(x, t) \in \Gamma \cap \Sigma$, suppose $\left(x+k e_{n}, t+\tau\right) \in \Gamma$. Then there exists a dimensional constant $K_{1}>0$ such that

$$
|k| \leq r^{5 / 4} \quad \text { if } \tau \in\left[0, \frac{r^{5 / 4}}{K_{1} M C_{0}}\right] .
$$

Proof. Due to Lemma 3.6, at any time $0 \leq t \leq t\left(x_{0}, r\right)$, we have

$$
h^{+}(x, t) \leq u^{+}(x, t) \leq C_{1} h^{+}\left(x-r^{5 / 4} e_{n}, t\right)
$$


and

$$
h^{-}(x, t) \leq u^{-}(x, t) \leq C_{1} h^{-}\left(x+r^{5 / 4} e_{n}, t\right),
$$

in $B_{r}\left(x_{0}\right)$, where the function $h:=h^{+}(\cdot, t)-h^{-}(\cdot, t)$ is harmonic in its positive and negative phases in $(1+r) \Omega_{t}(u)-(1-r) \Omega_{t}(u)$, and the domains $\Omega\left(h^{+}\right)$and $\Omega\left(h^{-}\right)$are both star-shaped with respect to $B_{r_{0}}(0)$.

Let us pick $\left(y_{0}, t_{0}\right) \in \Gamma \cap \Sigma$. Due to Proposition 4.3, (4-4) and the Harnack inequality for harmonic functions, we have

$$
\sup _{y \in B_{100^{5} / 4\left(y_{0}\right)}} u\left(y, t_{0}\right) \leq C C_{1} K M C_{0} r^{5 / 4},
$$

where $C$ is a dimensional constant. On the other hand, due to Lemma 3.1 and $t_{0}^{5} \leq r^{25 / 6}$, we have

$$
u\left(\cdot, t_{0}\right) \leq 0 \quad \text { in } B_{(1 / 2) r^{5 / 4}}\left(y_{0}+r^{5 / 4} e_{n}\right) .
$$

Let

$$
y_{1}:=y_{0}+r^{5 / 4} e_{n}, \quad C_{2}:=C C_{1} K M C_{0}, \quad r(t):=\frac{1}{2} r^{5 / 4}-C_{3}\left(t-t_{0}\right),
$$

where $C_{3}=C C_{2}$. Next we define $\phi(x, t)$ in the domain

$$
\Pi:=B_{2 r^{5 / 4}}\left(y_{1}\right) \times\left[t_{0}, t_{0}+\frac{r^{5 / 4}}{C_{3}}\right]
$$

such that

$$
\begin{cases}-\Delta \phi(\cdot, t)=0 & \text { in } B_{2 r^{5 / 4}}\left(y_{1}\right)-B_{r(t)}\left(y_{1}\right), \\ \phi=2 C_{2} r^{5 / 4} & \text { on } \partial B_{2 r^{5 / 4}}\left(y_{1}\right), \\ \phi=0 & \text { in } B_{r(t)}\left(y_{1}\right) .\end{cases}
$$

Then by (4-4)-(4-7), $u \prec \phi$ at $t=t_{0}$ in $\Pi$. Let $T_{0}$ be the first time when $u$ hits $\phi$ from below in $\Pi$. Since (4-6) also holds for any $(x, t) \in \Gamma \cap \Sigma$ in place of $\left(y_{0}, t_{0}\right)$, we have $u<\phi$ on the parabolic boundary of $\Pi \cap\left\{t_{0} \leq t \leq T_{0}\right\}$. On the other hand, if $C$ is chosen sufficiently large, then

$$
\frac{\phi_{t}}{|D \phi|}=C_{3} \geq|D \phi| \quad \text { on } \partial B_{r(t)}\left(y_{1}\right) \times\left[t_{0}, t_{1}:=t_{0}+\frac{r^{5 / 4}}{4 C_{3}}\right],
$$

and thus $\phi$ is a supersolution of (ST1). This and Lemma 2.11 applied to $u$ and $\phi$ in $\Pi$ yields a contradiction, and we conclude that $\Gamma(u)$ lies outside of $B_{\frac{1}{4} r^{5 / 4}}\left(y_{0}+r^{5 / 4} e_{n}\right)$ for $t_{0} \leq t \leq t_{1}$.

Similarly, by constructing a negative radial barrier and comparing it with $u$, one can show that $\Gamma(u)$ lies outside of $B_{\frac{1}{4}} r^{5 / 4}\left(y_{0}-r^{5 / 4} e_{n}\right)$ for $t_{0} \leq t \leq t_{1}$. This concludes the proof.

For $x_{0} \in \Gamma_{t_{0}}$, define

$$
t\left(x_{0}, r\right):=\min \left\{\frac{r^{2}}{u^{+}\left(x_{0}-r e_{n}, t_{0}\right)}, \frac{r^{2}}{u^{-}\left(x_{0}+r e_{n}, t_{0}\right)}\right\} .
$$

We now proceed to show our main result, Proposition 4.3. First we show Harnack-type inequalities for positive times. 
Lemma 4.5 (Harnack at later times). Fix $s \in\left[r^{5 / 4}, r\right]$. If $\left(y_{0}, t_{0}\right) \in \Gamma \cap \Sigma$, then

$$
u^{+}\left(y_{0}-s e_{n}, t_{0}\right) \geq c_{1} u^{+}\left(y_{0}-s e_{n}, t_{0}+\tau\right) \quad \text { and } \quad u^{-}\left(y_{0}+s e_{n}, t_{0}\right) \geq c_{1} u^{-}\left(y_{0}+s e_{n}, t_{0}+\tau\right)
$$

for $0 \leq \tau \leq t\left(y_{0}, s\right) / 2$ and $c_{1}>0$.

Proof. We will show the lemma for $u^{+}$, the statement for $u^{-}$follows via parallel arguments.

Step 1. Let $\left(y_{0}, t_{0}\right) \in \Gamma \cap \Sigma$ and let $s \in\left[r^{5 / 4}, r\right]$. Let $h^{+}$be given as in (4-4). Due to Lemma 3.3 and Lemma 3.4, we have

$$
h^{+}\left(y_{0}-2 r e_{n}, t_{1}\right) \leq u^{+}\left(y_{0}-2 r e_{n}, t_{1}\right) \leq C u^{+}\left(y_{0}-2 r e_{n}, t_{2}\right) \leq C h^{+}\left(y_{0}-\left(2 r+r^{5 / 4}\right) e_{n}, t_{2}\right)
$$

for $0 \leq t_{1}, t_{2} \leq t_{0}+t\left(y_{0}, r\right) / 2$. (Here note that $y_{0} \in B_{r}\left(x_{0}\right)$.) In particular

$$
u^{+}\left(y_{0}-2 r e_{n}, t\right) \leq C h^{+}\left(y_{0}-\left(2 r+r^{5 / 4}\right) e_{n}, t_{0}\right) \leq C_{1} h^{+}\left(y_{0}-2 r e_{n}, t_{0}\right)
$$

for $t \leq t_{0}+t\left(y_{0}, s\right) / 2$.

Step 2. Now let $v^{* *}$ solve $(\mathrm{ST} 1)$ in $\left(\mathbb{R}^{n}-(1-2 r) D_{t_{0}}\right) \times\left[t_{0}, t_{0}+t\left(y_{0}, s\right) / 2\right]$ with initial and boundary $\overline{\text { data } C_{2}} h^{+}\left(x-2 s e_{n}, t\right)$. Since $s \geq r^{5 / 4}$, (4-4) implies

$$
\Omega_{t}(u) \subset \Omega_{t_{0}}\left(v^{* *}\right) \subset \Omega_{t}\left(v^{* *}\right) \quad \text { in } B_{2 s}\left(y_{0}\right) \times\left[t_{0}, t_{0}+t\left(y_{0}, s\right) / 2\right] .
$$

Then by (4-9), (4-8) and (4-4),

$$
u^{+} \leq v^{* *} \quad \text { in } B_{s}\left(y_{0}\right) \times\left[t_{0}, t_{0}+t\left(y_{0}, s\right) / 2\right]
$$

if we choose $C_{2}$ as a multiple of $C_{1}$ by a dimensional constant. Moreover, due to the Harnack inequality for one-phase (ST1), one can conclude that

$$
\begin{aligned}
u^{+}\left(y_{0}-s e_{n}, t_{0}+\tau\right) & \leq v^{* *}\left(y_{0}-s e_{n}, t_{0}+\tau\right) \\
& \leq C v^{* *}\left(y_{0}-s e_{n}, t_{0}\right) \\
& =C C_{2} h^{+}\left(y_{0}-3 s e_{n}, t_{0}\right) \\
& \leq C_{3} h^{+}\left(y_{0}-s e_{n}, t_{0}\right) \leq C_{3} u^{+}\left(y_{0}-s e_{n}, t_{0}\right)
\end{aligned}
$$

for

$$
0 \leq \tau \leq \frac{s^{2}}{v^{* *}\left(y_{0}-s e_{n}, t_{0}\right)} \sim t\left(y_{0}, s\right) / 2 .
$$

Here the first inequality uses the fact $u^{+} \leq v^{* *}$, the second uses the Harnack inequality for $v^{* *}$, the third one uses the Harnack inequality for harmonic functions and the last one uses (4-4).

Lemma 4.6 (backward Harnack). Suppose that (4-3) holds up to time $t=T_{0} \leq t\left(x_{0}, r\right)$. If $\left(y_{0}, t_{0}\right) \in \Gamma$ and $t_{0} \leq T_{0}$, then for $0 \leq \tau \leq t\left(y_{0}, s\right) / 2$,

$$
u^{+}\left(y_{0}-s e_{n}, t_{0}\right) \leq C u^{+}\left(y_{0}-s e_{n}, t_{0}+\tau\right) \quad \text { and } \quad u^{-}\left(y_{0}+s e_{n}, t_{0}\right) \leq C u^{-}\left(y_{0}+s e_{n}, t_{0}+\tau\right),
$$

where $0 \leq s \leq r$ and $C$ is a universal constant. 
Proof. We will show the argument for $u^{+}$, due to the symmetric nature of the claim. The argument here will be similar to that of Lemma 3.4, replacing the initial data $u_{0}^{+}$and $u_{0}^{-}$(used in the construction of barriers) by $h^{+}\left(x, t_{0}\right)$ and $h^{-}\left(x, t_{0}\right)$ given in (4-4)-(4-5).

We consider a solution $v_{1}$ of (ST1) in

$$
\Pi:=(1+r) \Omega_{t_{0}} \times\left[t_{0}, t_{0}+t\left(y_{0}, s\right) / 2\right]
$$

with initial and lateral boundary data $C_{1} h^{-}$. Then $v_{1} \leq u$ in $\Pi$. Now let $v_{2}$ solve the heat equation in $\left\{v_{1}=0\right\} \times\left[t_{0}, t_{0}+t\left(y_{0}, s\right) / 2\right]$ with initial data

$$
v_{2}\left(\cdot, t_{0}\right)= \begin{cases}h^{+}\left(\cdot, t_{0}\right) & \text { in }\left\{v_{1}\left(\cdot, t_{0}\right)=0\right\}-(1-r)\left\{h^{+}\left(\cdot, t_{0}\right)>0\right\} \\ \tilde{h}(\cdot) & \text { in }(1-r)\left\{h^{+}\left(\cdot, t_{0}\right)>0\right\}\end{cases}
$$

where $\tilde{h}(\cdot)$ is a $C^{2}$ extension function of $h^{+}\left(\cdot, t_{0}\right)$ chosen so that $\tilde{h}(\cdot) \leq u^{+}\left(\cdot, t_{0}\right)$. The rest of the proof is the same as that of Lemma 3.4.

Next we show that in the unbalanced region, possibly forming at positive times, the fast regularization phenomena still holds. This lemma will be used in the proof of Proposition 4.3 to show that there cannot be a severe unbalance of flux in the initially balanced region $\Sigma$.

Lemma 4.7 (regularization in unbalanced region II). For a fixed $\left(x_{0}, t_{0}\right) \in \Gamma(u)$, suppose that

$$
u^{+}\left(x_{0}-r e_{n}, t_{0}\right) \geq M u^{-}\left(x_{0}+r e_{n}, t_{0}\right) \quad \text { or } u^{-}\left(x_{0}+r e_{n}, t_{0}\right) \geq M u^{+}\left(x_{0}-r e_{n}, t_{0}\right)
$$

for $M>M_{n}$, where $M_{n}$ is a dimensional constant. Then for $r \leq 1 / M_{n}$, there exists a dimensional constant $C>0$ such that

$$
\left|D u^{+}\right| \leq C \frac{u^{+}\left(x_{0}-r e_{n}, t_{0}\right)}{r} \quad \text { and } \quad\left|D u^{-}\right| \leq C \frac{u^{-}\left(x_{0}+r e_{n}, t_{0}\right)}{r}
$$

in $B_{r}\left(x_{0}\right) \times\left[t_{0}+t\left(x_{0}, r\right) / 2, t_{0}+t\left(x_{0}, r\right)\right]$.

Proof. The proof of this lemma is parallel to that of Proposition 3.7. We use the Harnack and backward Harnack inequalities (Lemmas 4.5 and 4.6) instead of Lemmas 3.3 and 3.4.

We are now ready to prove our main result, Proposition 4.3. Observe that (4-3) holds up to some $T_{0}>0$ by Lemma 4.2 and Lemma 3.3.

Proof of Proposition 4.3. Let $K$ be a sufficiently large dimensional constant such that $K \gg M$. Let us assume that (4-3) breaks down for $u^{+}$for the first time at $t=T_{0}$. Then

$$
\frac{u^{+}\left(z_{0}-s e_{n}, T_{0}\right)}{s}=K M C_{0}
$$

for some $\left(z_{0}, T_{0}\right) \in \Gamma \cap \Sigma$ and $r^{5 / 4} \leq s \leq r$. Let

$$
h=\sup \left\{h: \frac{u^{+}\left(z_{0}-k e_{n}, T_{0}\right)}{k} \geq M^{2} C_{0} \quad \text { for } s \leq k \leq h\right\} .
$$


Note that $h<r / 2$ due to Lemma 3.3 and the definition of $C_{0}$, and $h>2 s$ due to Lemma 3.6. By the definition of $h$ we have

$$
\frac{u^{+}\left(z_{0}-h e_{n}, T_{0}\right)}{h}=M^{2} C_{0}
$$

Let us find the largest time $t_{0}$ before $T_{0}$ such that for some $\left(y_{0}, t_{0}\right) \in \Gamma$

$$
T_{0}-t_{0}=\frac{t\left(y_{0}, h\right)}{2} \text { and } \quad \frac{y_{0}}{\left|y_{0}\right|}=\frac{z_{0}}{\left|z_{0}\right|} .
$$

Then Lemma 4.5 implies

$$
\frac{u^{+}\left(y_{0}-h e_{n}, t_{0}\right)}{h} \sim \frac{u^{+}\left(y_{0}-h e_{n}, T_{0}\right)}{h} \sim \frac{u^{+}\left(z_{0}-h e_{n}, T_{0}\right)}{h}=M^{2} C_{0} .
$$

Since $u^{+}\left(\cdot, t_{0}\right)$ and $u^{-}\left(\cdot, t_{0}\right)$ are comparable to harmonic functions (Lemma 3.6), a similar argument as in Lemma 4.1 implies that

$$
\frac{u^{-}\left(y_{0}+h e_{n}, t_{0}\right)}{h} \lesssim C_{0} \lesssim \frac{1}{M^{2}} \frac{u^{+}\left(y_{0}-h e_{n}, t_{0}\right)}{h} .
$$

Hence by Lemma 4.7, we have

$$
\left|D u^{+}\left(\cdot, T_{0}\right)\right| \sim M^{2} C_{0} \quad \text { in } B_{h}\left(y_{0}\right) .
$$

Since $B_{s}\left(z_{0}\right) \subset B_{h}\left(y_{0}\right)$, this would contradict (4-10) as $K \gg M$.

Due to Lemma 3.6, Proposition 4.3 and Corollary 4.4, we have shown that condition (A) holds and that the level sets of $u$ are close to a Lipschitz graph, and $\Gamma(u)$ is close to a Lipschitz graph in space and time (see the detailed description of this fact in the next section). However, we do not yet have sufficient control of the change of $u$ over time to verify the condition $\left(\mathrm{B}^{\prime}\right)$. We will therefore prove Theorem 1.1 by carrying out a modified argument, combining arguments from [Athanasopoulos et al. 1996; 1998] and [Choi et al. 2007; 2009].

\section{Further regularization based on flatness}

Let $u, \Gamma_{0}$ be as given in Theorem 1.1. Recall that $x_{0} \in \Gamma_{0}$ and $r>0$ are fixed, and they satisfy (4-1). Let $C_{0}$ be as given in (4-2) and $t\left(x_{0}, r\right)$ as given in (1-3).

Our goal is to prove the regularization of the free boundary after the time $t\left(x_{0}, r\right) / 2$ in $B_{r}\left(x_{0}\right)$. Define

$$
\Sigma_{r}\left(x_{0}\right):=B_{r}\left(x_{0}\right) \times\left[t\left(x_{0}, r\right) / 2, t\left(x_{0}, r\right)\right] \subset \Sigma .
$$

Let us briefly review the information we have on $u$ so far. As a result of Proposition 4.3, condition (A) holds up to

$$
t=t\left(x_{0}, r\right) \leq C r^{2-\alpha}<r^{3 / 4} \text {. }
$$

Also due to Lemma 3.6, our solution $u$ is $\epsilon$-monotone in $Q_{r}\left(x_{0}\right)$ with respect to a space cone $W_{x}\left(e_{n}, \theta_{0}\right)$ satisfying

$$
\left|\theta_{0}-\pi\right|=O(L),
$$


where $L$ is the Lipschitz constant of the initial domain $\Omega_{0}$ given by (1-1).

Moreover $Q_{r}\left(x_{0}\right) \subset \Sigma$, and thus Corollary 4.4 and Lemma 3.1 yield that the free boundary $\Gamma(u)$ is $r^{4 / 3}$-monotone in $Q_{r}\left(x_{0}\right)$ with respect to the time cone $W_{t}\left(e_{n}, \tan ^{-1}\left(1 / K_{1} M C_{0}\right)\right)$ and the space cone $W_{x}\left(e_{n}, \theta_{0}\right)$. Here $\theta_{0}$ is the angle corresponding to the Lipschitz constant of $\Gamma_{0}$, and $t\left(x_{0}, r\right)=r / C_{0}$.

On the other hand, by Lemma 3.3 and the definition of $C_{0}$,

$$
\frac{u\left(x_{0}-r e_{n}, \frac{1}{2} t\left(x_{0}, r\right)\right)}{C_{0} r} \sim 1 .
$$

Since $Q_{r}\left(x_{0}\right) \subset \Sigma$, Proposition 4.3 implies

$$
\frac{u(x, t)}{C_{0} r} \lesssim K M \quad \text { in } B_{r}\left(x_{0}\right) \times\left[t\left(x_{0}, r\right) / 2, t\left(x_{0}, r\right)\right] .
$$

The main difficulty in applying the method of [Athanasopoulos et al. 1996; 1998] lies in the fact that we cannot guarantee the $\epsilon$-monotonicity of the solution $u$ in the time variable (although we can obtain, as above, the $r^{4 / 3}$-monotonicity of the free boundary $\Gamma(u)$ ). To go around this difficulty, we will first use the parabolic scale to improve the regularity of the solution in space. Consider the function

$$
\bar{u}(x, t):=\frac{1}{C_{0} r} u\left(r x+x_{0}, r^{2} t+\frac{1}{2} t\left(x_{0}, r\right)\right) .
$$

In [Athanasopoulos et al. 1996; 1998], it was important that initially the time derivative of the solution was assumed to be controlled by the spatial derivative, i.e.,

$$
\left|u_{t}\right| \leq C\left(\left|D u^{+}\right|+\left|D u^{-}\right|\right) .
$$

Using (5-2) one can prove that the direction vectors

$$
\frac{D u^{+}}{\left|D u^{+}\right|}\left(-l e_{n}, t\right) \text { and } \frac{D u^{-}}{\left|D u^{-}\right|}\left(l e_{n}, t\right)
$$

do not change much for $0 \leq t \leq l$. This is pivotal in the regularization procedure since then $\Gamma(u)$ regularizes along the direction of the "common gain" obtained by those two direction vectors, the regularity of $\Gamma(u)$ then makes the above two vectors line up better in a smaller scale, which contributes to further regularization of $\Gamma(u)$ in a finer scale. In our case we do not know a priori that $\Gamma(u)$ is Lipschitz in either space or in time; in fact the Lipschitz continuity of $\Gamma(u)$ in time will be proved in the very last stage of Section 5 (see Theorem 5.7). Therefore, we do not have (5-2), and thus extra care is required to show that the spatial gradients $D u^{ \pm}$do not change their directions too rapidly.

In the following series of results, we will assume that $\bar{u}$ is given by (5-1). The lemmas and theorems will be proved to in the order they are stated, to improve the regularity of $\bar{u}$ in multiple steps.

Lipschitz continuity in space. First we prove that the $\epsilon$-monotonicity of $\Gamma(\bar{u})$ improves to Lipschitz continuity. Let $a=C_{0} r$. Then, in the domain $B_{1}(0) \times[-1 / a, 1 / a], \bar{u}(x, t)$ solves

$$
\begin{cases}\bar{u}_{t}-\Delta \bar{u}=0 & \text { in }\{\bar{u}>0\}, \\ V=a\left(\left|D \bar{u}^{+}\right|-\left|D \bar{u}^{-}\right|\right) & \text {on } \partial\{\bar{u}>0\} .\end{cases}
$$


Here note that

$$
r^{7 / 6} \leq r^{\alpha} \leq a \leq r^{\beta} \leq r^{5 / 6}
$$

In this scale, since $\bar{u}$ is caloric and $\Gamma(\bar{u})$ is $r^{1 / 3}$-close to a Lipschitz graph in space and time, it follows that so is $\bar{u}$ in $B_{1 / 2}(0) \times[-1 / a+1,1 / a]$.

Note that in above step we are losing a lot of information over time; $\Gamma(\bar{u})$ is in fact $r^{1 / 3}$-close to a Lipschitz graph moving very slowly in time, but this does not guarantee that $\bar{u}$ also changes slowly in time.

We then follow the iteration process in Lemma 7.2 of [Athanasopoulos et al. 1996] to show this:

Lemma 5.1. If $r$ is sufficiently small, then there exist $0<c, d<1 / 2$ such that $\bar{u}$ is $\lambda r^{1 / 3}$-monotone in the cone of directions $W_{x}\left(\theta_{x}-r^{d}, e_{n}\right)$ and $W_{t}\left(\theta_{t}-r^{d}, v\right)$ in the domain $B_{1-r^{c}}(0) \times\left[\left(-1+r^{c}\right) / a, 1 / a\right]$.

One can then iterate above lemma to improve the $\epsilon$-monotonicity to full monotonicity, and state the result in terms of $\bar{u}$ :

Lemma 5.2. $\bar{u}$ is fully monotone in $B_{1 / 2}(0) \times[0,1 / a]$ for the cone

$$
\mathscr{C}_{1}:=W_{x}\left(\theta_{x}-r^{d}, e_{n}\right) \cup W_{t}\left(\theta_{t}-r^{d}, v\right)
$$

for some constant $0<d<1 / 2$.

Regularity in time away from the free boundary. Now we suppose that $\bar{u}$ is Lipschitz in space and time. Then in particular, we have the Lipschitz regularity of $u$ in space (and very weak Lipschitz regularity of $u$ in time). We are interested in proving the following type of statement:

Lemma 5.3 (enlargement for the cone of monotonicity). There exists $\lambda>0$ such that if $\bar{u}$ is Lipschitz with respect to the cone of monotonicity $\Lambda_{x}\left(e_{n}, \theta_{0}\right)$ in $B_{1}(0) \times[-1 / a, 1 / a]$, then in the half domain $B_{1 / 2}(0) \times[-1 /(2 a), 1 /(2 a)], \bar{u}$ is Lipschitz with respect to the cone of monotonicity $\Lambda_{x}\left(v,(1+\lambda) \theta_{0}\right)$ with some unit vector $v$.

To prove the enlargement of the cone, we take a closer look at the change of $\bar{u}$ over time, in the interior region. More precisely, we need the following lemma, which follows the approach taken in [Choi et al. 2007; 2009].

Lemma 5.4. We have

$$
\left|\bar{u}_{t}\right| \leq a|D \bar{u}|^{2} \leq C a \quad \text { in }\left[B_{1 / 2}\left(e_{n}\right) \cup B_{1 / 2}\left(-e_{n}\right)\right] \times[-1 /(2 a), 1 /(2 a)],
$$

where $C$ is a dimensional constant.

Proof. Step 1. The proof is similar to that of Lemma 8.3 of [Choi et al. 2009]. Note that $\bar{u}_{t}$ is a caloric function in $\Omega^{+}(\bar{u})$ and $\Omega^{-}(\bar{u})$. Let us prove the lemma for $\bar{u}^{+}$, since parallel arguments apply to $\bar{u}^{-}$.

Step 2. We divide $\bar{u}_{t}$ into two parts. More precisely, let

$$
\bar{u}_{t}=v_{1}+v_{2}
$$


where both functions $v_{1}$ and $v_{2}$ are caloric in $\Omega^{+}(\bar{u}), v_{1}$ has the initial data zero and the boundary data $a\left|D \bar{u}^{+}\right|\left(\left|D \bar{u}^{+}\right|-\left|D \bar{u}^{-}\right|\right)$on $\Gamma(\bar{u})$, and $v_{2}$ has the initial data $\bar{u}_{t}(\cdot,-1 / a)$ and the boundary data zero on $\Gamma(\bar{u})$.

Step 3. For $v_{1}$, we need to use the absolute continuity of the caloric measure with respect to the harmonic measure, as well as the Lipschitz continuity of the free boundary. We proceed as in Lemma 8.3 of [Choi et al. 2007]. Note that we have

$$
\left|D \bar{u}^{+}\right| \sim\left|D \bar{u}^{-}\right| \sim 1
$$

in $\left[B_{1 / 2}\left(e_{n}\right) \cup B_{1 / 2}\left(-e_{n}\right)\right] \times[-1 / a, 1 / a]$ : this follows from the assumption in (4-1), and Lemmas 3.3 and 3.4. Therefore we can proceed as in Lemma 8.3 of [Choi et al. 2007] to obtain

$$
v_{1}(x, t) \leq a \int_{\Gamma(\bar{u}) \cap\{-1 / a \leq s \leq t\}}\left|D \bar{u}^{+}\right|^{2} d \omega^{(x, t)} \leq a|D \bar{u}|^{2}(x, t),
$$

where $\omega^{(x, t)}$ is the caloric measure for $\Omega(\bar{u})$, and

$$
v_{1}(x, t) \geq a \int_{\Gamma(\bar{u}) \cap\{-1 / a \leq s \leq t\}}-\left|D \bar{u}^{-}\right|^{2} d \omega^{(x, t)} \geq-a|D \bar{u}|^{2}(x, t) .
$$

Step 4. As for $v_{2}$, we conclude that it must be smaller than a caloric function solved in the whole domain with the absolute value of its initial data. The advantage is that then we can use the heat kernel. Note that the initial data is given at $t=-1 / a$ and has compact support. The initial data is given by $v_{t} \leq(C / a) v_{e_{n}}$, where $v_{e_{n}}(x, t)$ is comparable to the derivative of a harmonic function in a Lipschitz domain.

Therefore the heat kernel representation is given as

$$
\frac{1}{(t+1 / a)^{n / 2+1}} \int\left|x_{n}-y_{n}\right| \exp ^{-|x-y|^{2} /(t+1 / a)} v(y,-1 / a) d y .
$$

Since $t \in[0,1 / a]$ and $k \exp ^{-a k^{2}} \leq C \exp ^{-(a / 2) k^{2}}$, we get the effect of $O(a)$.

Further regularity in space. Now that we have sufficient information on the change of $u$ over time, we change the scale following the one introduced in (1-4), and consider the function

$$
v(x, t):=\frac{1}{C_{0} r} u\left(r x+x_{0}, \frac{r}{C_{0}} t+1\right) .
$$

Note that $C_{0}=r^{-1} c\left(x_{0}, r\right)$, and thus $v$ coincides with $\tilde{u}$ defined in (1-4) with the choice of $c=r C_{0}$.

Due to the previous results, this function is Lipschitz continuous, in space and time, away from the free boundary. The following lemma suggests that the cone of monotonicity improves away from the free boundary, as we look at smaller scales. The proof is parallel to that of Lemma 8.4 in [Athanasopoulos et al. 1998].

Lemma 5.5. Let $v$ given by (5-3). Suppose that there exist constants $\delta>0$ and $0 \leq A \leq B, \mu:=B-A$, such that

$$
\alpha\left(D v,-e_{n}\right) \leq \delta \quad \text { and } \quad A \leq \frac{v_{t}}{-e_{n} \cdot D v} \leq B
$$


in $B_{1 / 6}\left(-\frac{3}{4} e_{n}\right) \times(-\delta / \mu, \delta / \mu)$ with $\delta / \mu<r$. Then there exist a unit vector $v \in \mathbb{R}^{n}$ and positive constants $r_{0}, b_{0}<1$ depending only on $A, B$ and $n$ such that

$$
\alpha(D v(x, t), v) \leq b_{0} \delta \quad \text { in } B_{1 / 8}\left(-\frac{3}{4} e_{n}\right) \times\left(-r_{0} \frac{\delta}{\mu}, r_{0} \frac{\delta}{\mu}\right) .
$$

Now we can proceed as in Section 6 of [Choi et al. 2009] to obtain further regularity, using Lemma 5.4 instead of the uniform upper bound on $|D u|$ up to the free boundary.

Theorem 5.6. $\Gamma(v)$ is $C^{1}$ in space in $Q_{1 / 2}$. In particular, there exist dimensional constants $l_{0}, C_{0}>0$ such that for a free boundary point $\left(x_{0}, t_{0}\right) \in \Gamma(v), \Gamma(v) \cap\left(B_{2^{-l}}\left(x_{0}\right) \times\left[t_{0}-2^{-l}, t_{0}+2^{-l}\right]\right.$ is a Lipschitz graph in space with Lipschitz constant less than $C_{0} / l$ if $l \geq l_{0}$.

Regularity in time up to the free boundary. Lastly, proceeding as in Sections 7-8 of [Choi et al. 2009] yields the differentiability of $\Gamma(v)$ in time. The main step in the argument is the following proposition: the statement and its proof is parallel to those of Theorem 7.2 in [Choi et al. 2009] and the blow-up argument as in Section 8 of [Choi et al. 2009]:

Theorem 5.7. $\Gamma(v)$ is differentiable in space and time. More precisely there exist dimensional constants $l_{0}>0$ and $1<\gamma<2$ such that for $\left(x_{0}, t_{0}\right) \in \Gamma(v) \cap Q_{1}$, if $l>l_{0}$ then $\Gamma(v) \cap\left(B_{2^{-l}}\left(x_{0}\right) \times\left[t_{0}-2^{-l}, t_{0}+2^{-l}\right]\right.$ is a Lipschitz graph in space with Lipschitz constant less than $l^{-\gamma}$, and Lipschitz graph in time with Lipschitz constant less than $l^{-1 / 3}$.

\section{Corollary 5.8 .}

$$
C^{-1} \leq\left|D v^{+}\right|(x, t) \leq C, \quad C^{-1} \leq \frac{\left|D v^{-}\right|(x, t)}{v\left(-e_{n}, t\right)} \leq C
$$

in $Q_{1 / 2}$, where $C=C(n)$.

\section{General case: solutions with locally Lipschitz initial data}

In this section, we present how to extend the result of the main theorem to solutions with locally Lipschitz initial data. Our setting is as follows. Suppose $\Omega_{0}$ is a bounded region in $B_{R}(0)$. Suppose $u$ is a solution of (ST2) with $u_{0} \geq-1, u_{0}=-1$ in $B_{R}(0)$ and $u_{0} \leq M_{0}$. Further suppose that $\Omega_{0}$ is locally Lipschitz, that is, for any $x_{0} \in \Gamma_{0}, \Gamma_{0} \cap B_{1}\left(x_{0}\right)$ is Lipschitz with a Lipschitz constant $L \leq L_{n}$.

Let the initial data $u_{0}$ solve $\Delta u_{0}=0$ in $B_{1}\left(x_{0}\right)$. Then we claim that the parallel statements as in Theorem 1.1 hold in $B_{2 d_{0}}\left(x_{0}\right) \times\left[t\left(x_{0}, d_{0}\right) / 2, t\left(x_{0}, d_{0}\right)\right]$, where $d_{0}$ is a constant depending on $n$ and $M_{0}$. More precisely:

Theorem 6.1. Suppose $u$ is a solution of (ST2) with initial data $u_{0}$ such that $-1 \leq u_{0} \leq M_{0}$. Further suppose that for $x_{0} \in \Gamma_{0}, \Gamma_{0} \cap B_{1}\left(x_{0}\right)$ is Lipschitz with a Lipschitz constant $L \leq L_{n}$ and $\Delta u_{0}=0$ in the positive and negative phases of $u_{0}$ in $B_{1}\left(x_{0}\right)$. Then there exists a constant $d_{0}>0$ depending on $n$ and $M_{0}$ such that $(a)$ and $(b)$ of Theorem 1.1 hold for $u$ and $d \leq d_{0}$.

The proof of the above theorem is parallel to that of Theorem 1.1 in Section 5, after proving the following lemma. 


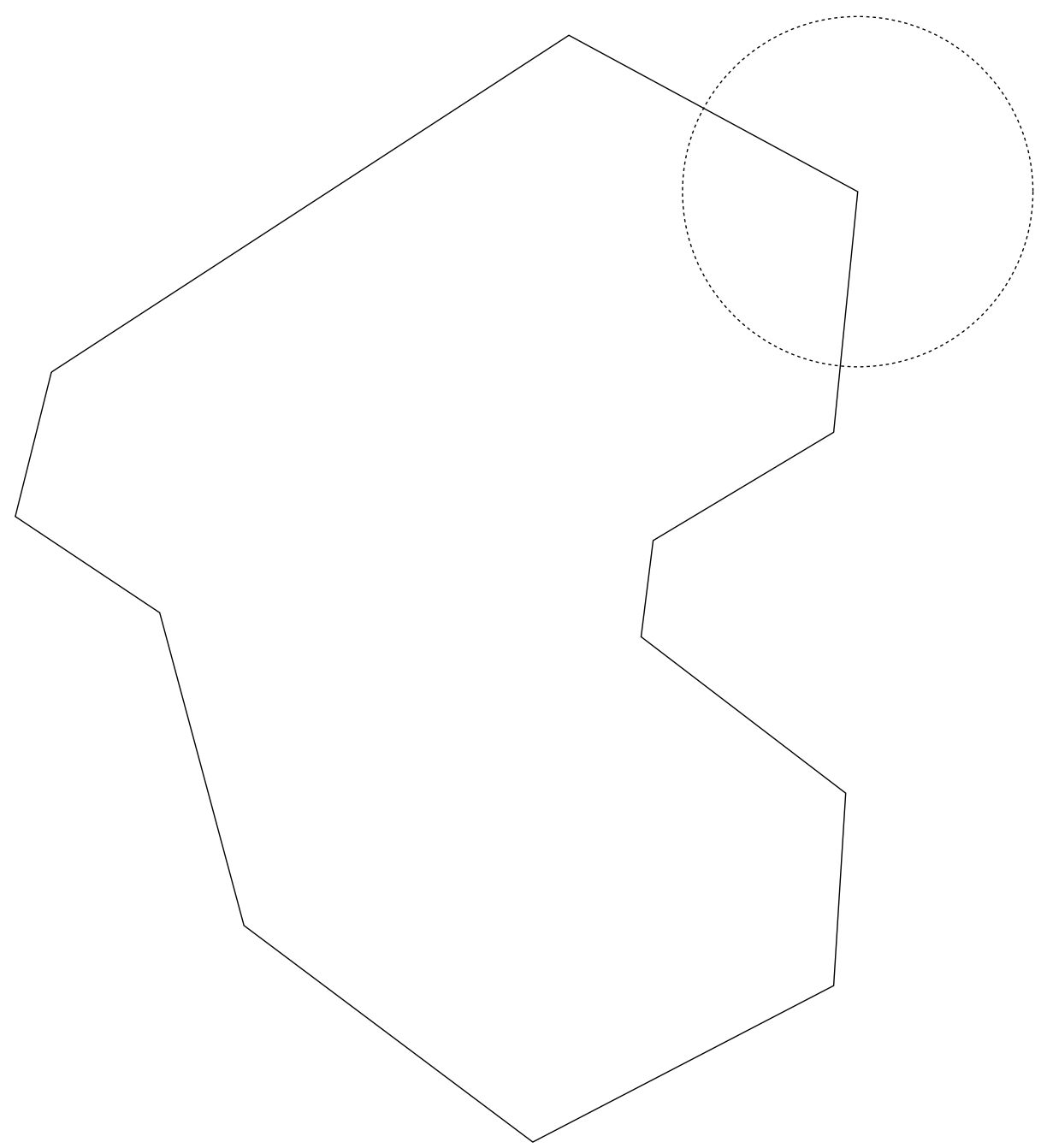

Figure 4. Locally Lipschitz initial domain.

Lemma 6.2. There exists a solution $v$ of (ST2) with star-shaped initial data such that the level sets of $u$ and $v$ are $\epsilon d_{0}$-close to each other in $B_{2 d_{0}}\left(x_{0}\right)$ up to the time $t\left(x_{0}, d_{0} ; u\right)$, where $d_{0}>0$ is sufficiently small. In particular, $u$ and $\Gamma(u)$ are $\epsilon$-monotone in a cone of $W_{x}$ and $W_{t}$ in $B_{2 d_{0}}\left(x_{0}\right) \times\left[t\left(x_{0}, d_{0}\right) / 2, t\left(x_{0}, d_{0}\right)\right]$.

Even though our equation is nonlocal, the behavior in a far-away region would not affect much the behavior of the solution in the unit ball, if the solution behaves "reasonably" outside the unit ball. For example, in the star-shaped case, we know at least that the free boundary is almost locally Lipschitz at each time. In the locally Lipschitz case, we control the solution by putting an upper bound $M_{0}$ on the initial data $u_{0}$. We will argue that in a sufficiently small subregion of $B_{1}\left(x_{0}\right) \times[0,1]$, the solution is mostly determined by the local initial data in $B_{1}\left(x_{0}\right)$. The perturbation method in the proof of Lemma 2.4 in [Choi et al. 2007] will be adopted here. Write $B_{1}\left(x_{0}\right)=B_{1}$. 
Step 1. Construct a star-shaped region $\Omega^{\prime} \subset B_{R}(0)$ such that:

(a) $\Omega^{\prime} \cap B_{1}=\Omega_{0} \cap B_{1}$.

(b) $\Omega^{\prime}$ is star-shaped with respect to every $x \in K \subset \Omega^{\prime}$ for a sufficiently large ball $K$.

Let $v_{0}^{+}$be the harmonic function in $\Omega^{\prime}-K$ with boundary data 1 on $\partial K$, and 0 on $\partial \Omega^{\prime}$. Next, let $v_{0}^{-}$ be the harmonic function in $B_{R}(0)-\Omega^{\prime}$ with boundary data 1 on $\partial B_{R}(0)$, and 0 on $\partial \Omega^{\prime}$. Let $B_{2}$ be a concentric ball in $B_{1}$ with the radius of $\epsilon^{k_{0}}$, i.e.,

$$
B_{2}=B_{\epsilon^{k_{0}}}\left(x_{0}\right) \subset B_{1}\left(x_{0}\right)=B_{1} .
$$

Let $k_{0}$ be sufficiently large. Then by Lemma 2.7 , a normalization of $v_{0}^{ \pm}$by a suitable constant multiple yields that for any $x \in B_{2}$,

$$
1-\epsilon \leq \frac{u_{0}(x)}{v_{0}(x)} \leq 1+\epsilon
$$

Let $v$ solve (ST2) with initial data $v_{0}=v_{0}^{+}-v_{0}^{-}$. Then Theorem 1.1 applies for $v$ since $v_{0}$ is star-shaped with respect to $K$.

For the proof of the claim, we will find a sufficiently small $d_{0}$ such that $v$ is $\epsilon d_{0}$-close to $u$ in $B_{2 d_{0}}\left(x_{0}\right)$ up to the time $t\left(x_{0}, d_{0}\right)$. More precisely, we will construct a supersolution $w_{1}$ and a subsolution $w_{2}$ of (ST2) such that in some small ball $B_{h}\left(x_{0}\right)$, we have

$$
w_{2} \leq u \leq w_{1}
$$

and the level sets of $w_{1}$ and $w_{2}$ are $h \epsilon$ close to the level sets of $v$.

Step 2. Let $k_{1}$ and $k_{2}$ be large constants which will be determined later. Define

$$
H^{ \pm}:=\left(\Gamma_{0}(v) \pm \epsilon^{k_{0}+k_{1}} e_{n}\right) \cap B_{2} .
$$

Let

$$
d_{0}:=\epsilon^{k_{0}+k_{1}+k_{2}}
$$

and let $t\left(d_{0}\right):=t\left(x_{0}, d_{0} ; v\right)=t\left(x_{0}, d_{0} ; u\right)$. First note that

$$
t\left(d_{0}\right) \geq d_{0}^{2-\beta} \geq \epsilon^{7\left(k_{0}+k_{1}+k_{2}\right) / 6} .
$$

Hence for $v$ to be almost harmonic in a scale much larger than $\epsilon^{k_{0}+k_{1}}$, we need $\sqrt{t\left(d_{0}\right)}>\epsilon^{k_{0}}$, i.e.,

$$
7\left(k_{0}+k_{1}+k_{2}\right) / 12<k_{0} .
$$

Observe that by the construction of $H^{ \pm}$and $d_{0}$,

$$
\sqrt{t\left(d_{0}\right)} \gg \operatorname{radius}\left(B_{2}\right) \gg \operatorname{dist}\left(H^{ \pm}, \Gamma_{0}\right) \gg \max _{x \in \Gamma_{t} \cap B_{2}, 0 \leq t \leq t\left(d_{0}\right)} \operatorname{dist}\left(x, \Gamma_{0}\right),
$$

where the last inequality follows from Lemma 2.12 if we choose $k_{2} \geq 2 k_{1}$. If $k_{2}$ is sufficiently large, then one can prove from the last inequality of (6-2) and the bound on $v_{t}$ that

$$
1-\epsilon \leq \frac{|v(x, t)|}{\left|v_{0}(x)\right|}=\frac{|v(x, t)|}{\left|u_{0}(x)\right|} \leq 1+\epsilon \quad \text { on } H^{ \pm} \times\left[0, t\left(d_{0}\right)\right] .
$$


Step 3. We do have an estimate, Lemma 2.12, on how far the boundaries move away for the local one-phase case. If we take the one-phase versions with initial data $u_{0}^{+}$and $u_{0}^{-}$, and compare with $u$, then we obtain that $\Gamma(u) \cap B_{2}$ stays in the $d_{0}^{(2-\alpha) /(2-\beta)}$-neighborhood of $\Gamma_{0}(u) \cap B_{2}$ up to the time $t\left(d_{0}\right)=t\left(x_{0}, d_{0}\right)$. In other words, the free boundary of $u$ moves less than $d_{0}^{5 / 7}$ in $B_{2}$ up to the time $t\left(d_{0}\right)$.

Now we let $S$ be the region between $H^{+}$and $H^{-}$. To construct a subsolution (or supersolution) in $\mathrm{S}$, we take the fixed boundary data $(1-\epsilon) v_{0}(x)$ on $\mathrm{H}^{-}$(or $\mathrm{H}^{+}$), and $(1+\epsilon) v_{0}(x)$ on $\mathrm{H}^{+}$(or $\mathrm{H}^{-}$). To control the effect from the side $\partial B_{2} \cap S$, we bend the free boundary $\Gamma_{t}(v)$ by $d_{0}^{5 / 7}$ on each side of $\partial B_{2} \cap S$, using the conformal mapping $\hat{\Phi}$ (or $\breve{\Phi}$ ). (See Section 4 of for the definitions of $\hat{\Phi}$ and $\breve{\Phi}$.) More precisely, we bend the free boundary of $v$ downward (or upward) using the conformal map $\hat{\Phi}$ (or $\breve{\Phi}$ ), and solve the heat equation in there. Then similar arguments as in Lemmas 4.1 and 4.3 of [Choi and Kim 2010] yield that the solution is still (almost) a supersolution, and it stays close to the original solution.

\section{References}

[Alt et al. 1984] H. W. Alt, L. A. Caffarelli, and A. Friedman, "Variational problems with two phases and their free boundaries", Trans. Amer. Math. Soc. 282:2 (1984), 431-461. MR 85h:49014 Zbl 0844.35137

[Athanasopoulos et al. 1996] I. Athanasopoulos, L. Caffarelli, and S. Salsa, "Caloric functions in Lipschitz domains and the regularity of solutions to phase transition problems", Ann. of Math. (2) 143:3 (1996), 413-434. MR 97e:35074 Zbl 0853.35049

[Athanasopoulos et al. 1998] I. Athanasopoulos, L. A. Caffarelli, and S. Salsa, "Phase transition problems of parabolic type: Flat free boundaries are smooth", Comm. Pure Appl. Math. 51:1 (1998), 77-112. MR 99b:35220 Zbl 0924.35197

[Caffarelli 1987] L. A. Caffarelli, "A Harnack inequality approach to the regularity of free boundaries, I: Lipschitz free boundaries are $C^{1, \alpha}$, Rev. Mat. Iberoamericana 3:2 (1987), 139-162. MR 90d:35306 Zbl 0676.35085

[Caffarelli and Cabré 1995] L. A. Caffarelli and X. Cabré, Fully nonlinear elliptic equations, Amer. Math. Soc. Coll. Publ. 43, Amer. Math. Soc., Providence, RI, 1995. MR 96h:35046 Zbl 0834.35002

[Caffarelli and Salsa 2005] L. Caffarelli and S. Salsa, A geometric approach to free boundary problems, Graduate Studies in Mathematics 68, American Mathematical Society, Providence, RI, 2005. MR 2006k:35310 Zbl 1083.35001

[Choi and Kim 2006] S. Choi and I. Kim, "Waiting time phenomena of the Hele-Shaw and the Stefan problem", Indiana Univ. Math. J. 55:2 (2006), 525-551. MR 2007h:35368 Zbl 05034334

[Choi and Kim 2010] S. Choi and I. C. Kim, "Regularity of one-phase Stefan problem near Lipschitz initial data", Amer. J. Math. 132:6 (2010), 1693-1727. MR 2012h:35384 Zbl 1231.35318

[Choi et al. 2007] S. Choi, D. Jerison, and I. Kim, "Regularity for the one-phase Hele-Shaw problem from a Lipschitz initial surface", Amer. J. Math. 129:2 (2007), 527-582. MR 2008d:35238 Zbl 1189.35384

[Choi et al. 2009] S. Choi, D. Jerison, and I. Kim, "Local regularization of the one-phase Hele-Shaw flow", Indiana Univ. Math. J. 58:6 (2009), 2765-2804. MR 2011c:35628 Zbl 1190.35229

[Dahlberg 1979] B. E. J. Dahlberg, "Harmonic functions in Lipschitz domains", pp. 313-322 in Harmonic analysis in Euclidean spaces (Williamstown, MA), vol. 1, edited by G. Weiss and S. Wainger, Proc. Sympos. Pure Math. 35, American Mathematical Society, Providence, RI, 1979. MR 80c:31002 Zbl 0425.31009

[Fabes et al. 1984] E. B. Fabes, N. Garofalo, and S. Salsa, "Comparison theorems for temperatures in noncylindrical domains", Atti Accad. Naz. Lincei Rend. Cl. Sci. Fis. Mat. Natur. (8) 77:1-2 (1984), 1-12. MR 88i:35069 Zbl 0625.35007

[Götz and Zaltzman 1991] I. G. Götz and B. B. Zaltzman, "Nonincrease of mushy region in a nonhomogeneous Stefan problem", Quart. Appl. Math. 49:4 (1991), 741-746. MR 92h:80011 Zbl 0756.35119

[Jerison and Kenig 1982] D. S. Jerison and C. E. Kenig, "Boundary behavior of harmonic functions in nontangentially accessible domains", Adv. in Math. 46:1 (1982), 80-147. MR 84d:31005b Zbl 0514.31003

[Kim and Požár 2011] I. C. Kim and N. Požár, "Viscosity solutions for the two-phase Stefan problem", Comm. Partial Differential Equations 36:1 (2011), 42-66. MR 2012b:35376 Zbl 1216.35181 
[Meirmanov 1992] A. M. Meirmanov, The Stefan problem, de Gruyter Expositions in Mathematics 3, Walter de Gruyter, Berlin, 1992. MR 92m:35282 Zbl 0751.35052

[Oleinik et al. 1993] O. Oleinik, M. Primicerio, and E. Radkevich, "Stefan-like problems", Meccanica 28 (1993), 129-143. Zbl 0786.76091

[Rogers and Berger 1984] J. C. W. Rogers and A. E. Berger, "Some properties of the nonlinear semigroup for the problem $u_{t}-\Delta f(u)=0 "$, Nonlinear Anal. 8:8 (1984), 909-939. MR 86b:35075 Zbl 0557.35129

Received 6 Feb 2011. Revised 12 Oct 2011. Accepted 15 Jan 2012.

Sunhi Choi: Department of Mathematics, University of Arizona, Tucson, AZ 85721, United States

schoi@math.arizona.edu

Inwon KIM: Department of Mathematics, UCLA, Los Angeles, CA 90095, United States

ikim@math.ucla.edu 


\title{
Analysis \& PDE
}

\author{
msp.berkeley.edu/apde
}

EDITORS

EDITOR-IN-CHIEF

Maciej Zworski

University of California

Berkeley, USA

BOARD OF EDITORS

\begin{tabular}{|c|c|c|c|}
\hline Michael Aizenman & $\begin{array}{l}\text { Princeton University, USA } \\
\text { aizenman@math.princeton.edu }\end{array}$ & Nicolas Burq & $\begin{array}{l}\text { Université Paris-Sud 11, France } \\
\text { nicolas.burq@math.u-psud.fr }\end{array}$ \\
\hline Luis A. Caffarelli & $\begin{array}{l}\text { University of Texas, USA } \\
\text { caffarel@math.utexas.edu }\end{array}$ & un-Yung Alice Chang & $\begin{array}{l}\text { Princeton University, USA } \\
\text { chang@math.princeton.edu }\end{array}$ \\
\hline Michael Christ & $\begin{array}{l}\text { University of California, Berkeley, USA } \\
\text { mchrist@math.berkeley.edu }\end{array}$ & Charles Fefferman & $\begin{array}{l}\text { Princeton University, USA } \\
\text { cf@math.princeton.edu }\end{array}$ \\
\hline Ursula Hamenstaedt & $\begin{array}{l}\text { Universität Bonn, Germany } \\
\text { ursula@math.uni-bonn.de }\end{array}$ & Nigel Higson & $\begin{array}{l}\text { Pennsylvania State Univesity, USA } \\
\text { higson@ math.psu.edu }\end{array}$ \\
\hline Vaughan Jones & $\begin{array}{l}\text { University of California, Berkeley, USA } \\
\text { vfr@math.berkeley.edu }\end{array}$ & Herbert Koch & $\begin{array}{l}\text { Universität Bonn, Germany } \\
\text { koch@math.uni-bonn.de }\end{array}$ \\
\hline Izabella Laba & $\begin{array}{l}\text { University of British Columbia, Canada } \\
\text { ilaba@math.ubc.ca }\end{array}$ & Gilles Lebeau & $\begin{array}{l}\text { Université de Nice Sophia Antipolis, France } \\
\text { lebeau@unice.fr }\end{array}$ \\
\hline László Lempert & $\begin{array}{l}\text { Purdue University, USA } \\
\text { lempert@math.purdue.edu }\end{array}$ & Richard B. Melrose & $\begin{array}{l}\text { Massachussets Institute of Technology, USA } \\
\text { rbm@math.mit.edu }\end{array}$ \\
\hline Frank Merle & $\begin{array}{l}\text { Université de Cergy-Pontoise, France } \\
\text { Frank.Merle@u-cergy.fr }\end{array}$ & William Minicozzi II & $\begin{array}{l}\text { Johns Hopkins University, USA } \\
\text { minicozz@ math.jhu.edu }\end{array}$ \\
\hline Werner Müller & $\begin{array}{l}\text { Universität Bonn, Germany } \\
\text { mueller@math.uni-bonn.de }\end{array}$ & Yuval Peres & $\begin{array}{l}\text { University of California, Berkeley, USA } \\
\text { peres@stat.berkeley.edu }\end{array}$ \\
\hline Gilles Pisier & $\begin{array}{l}\text { Texas A\&M University, and Paris } 6 \\
\text { pisier@math.tamu.edu }\end{array}$ & Tristan Rivière & $\begin{array}{l}\text { ETH, Switzerland } \\
\text { riviere@math.ethz.ch }\end{array}$ \\
\hline Igor Rodnianski & $\begin{array}{l}\text { Princeton University, USA } \\
\text { irod@math.princeton.edu }\end{array}$ & Wilhelm Schlag & $\begin{array}{l}\text { University of Chicago, USA } \\
\text { schlag@math.uchicago.edu }\end{array}$ \\
\hline Sylvia Serfaty & $\begin{array}{l}\text { New York University, USA } \\
\text { serfaty@ cims.nyu.edu }\end{array}$ & Yum-Tong Siu & $\begin{array}{l}\text { Harvard University, USA } \\
\text { siu@math.harvard.edu }\end{array}$ \\
\hline Terence Tao & $\begin{array}{l}\text { University of California, Los Angeles, USA } \\
\text { tao@math.ucla.edu }\end{array}$ & A Michael E. Taylor & $\begin{array}{l}\text { Univ. of North Carolina, Chapel Hill, USA } \\
\text { met@math.unc.edu }\end{array}$ \\
\hline Gunther Uhlmann & $\begin{array}{l}\text { University of Washington, USA } \\
\text { gunther@math.washington.edu }\end{array}$ & András Vasy & $\begin{array}{l}\text { Stanford University, USA } \\
\text { andras@math.stanford.edu }\end{array}$ \\
\hline Virgil Voiculescu & $\begin{array}{l}\text { University of California, Berkeley, USA } \\
\text { dvv@math.berkeley.edu }\end{array}$ & Steven Zelditch & $\begin{array}{l}\text { Northwestern University, USA } \\
\text { zelditch@math.northwestern.edu }\end{array}$ \\
\hline
\end{tabular}

PRODUCTION

production@msp.org

Silvio Levy, Scientific Editor

Sheila Newbery, Senior Production Editor

See inside back cover or msp.berkeley.edu/apde for submission instructions.

The subscription price for 2012 is US \$140/year for the electronic version, and \$240/year for print and electronic. Subscriptions, requests for back issues from the last three years and changes of subscribers address should be sent to Mathematical Sciences Publishers, Department of Mathematics, University of California, Berkeley, CA 94720-3840, USA.

Analysis \& PDE, at Mathematical Sciences Publishers, Department of Mathematics, University of California, Berkeley, CA 94720-3840 is published continuously online. Periodical rate postage paid at Berkeley, CA 94704, and additional mailing offices.

APDE peer review and production are managed by EditFLOW ${ }^{\mathrm{TM}}$ from Mathematical Sciences Publishers.

PUBLISHED BY

mathematical sciences publishers

http://msp.org/

A NON-PROFIT CORPORATION

Typeset in IATEX

Copyright $(2012$ by Mathematical Sciences Publishers 


\section{ANALYSIS \& PDE}

\section{Volume $5 \quad$ No. $5 \quad 2012$}

An inverse problem for the wave equation with one measurement and the pseudorandom 887 source

TAPio Helin, Matti Lassas and LAURi OKSANEN

Two-dimensional nonlinear Schrödinger equation with random radial data YU DENG

Schrödinger operators and the distribution of resonances in sectors

TANYA J. CHRISTIANSEN

Weighted maximal regularity estimates and solvability of nonsmooth elliptic systems, II

PASCAL AUSCHER and ANDREAS RosÉN

The two-phase Stefan problem: regularization near Lipschitz initial data by phase dynamics 1063 SUNHI CHOI and INWON KIM

$C^{\infty}$ spectral rigidity of the ellipse

HAMID HeZARI and STEVE ZELDITCH

A natural lower bound for the size of nodal sets

HAMID HEZARI and CHRISTOPHER D. SOGgE

Effective integrable dynamics for a certain nonlinear wave equation

PATRICK GÉRARD and SANDRINE GRELLIER

Nonlinear Schrödinger equation and frequency saturation 